\title{
2014s-17 \\ Identification-robust inference for endogeneity parameters in linear structural models
}

Firmin Doko Tchatoka, Jean-Marie Dufour

\begin{tabular}{c}
\hline Série Scientifique \\
Scientific Series
\end{tabular}

Montréal

Février 2014/February 2014

(C) 2014 Firmin Doko Tchatoka, Jean-Marie Dufour. Tous droits réservés. All rights reserved. Reproduction partielle permise avec citation du document source, incluant la notice (C).

Short sections may be quoted without explicit permission, if full credit, including (C) notice, is given to the source.
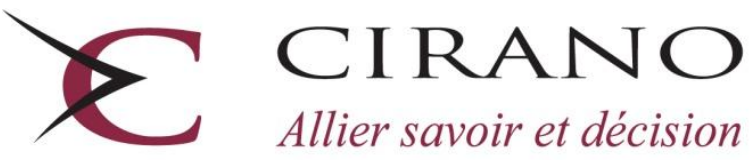

Allier savoir et décision

Centre interuniversitaire de recherche en analyse des organisations 


\section{CIRANO}

Le CIRANO est un organisme sans but lucratif constitué en vertu de la Loi des compagnies du Québec. Le financement de son infrastructure et de ses activités de recherche provient des cotisations de ses organisations-membres, d'une subvention d'infrastructure du Ministère de l'Enseignement supérieur, de la Recherche, de la Science et de la Technologie, de même que des subventions et mandats obtenus par ses équipes de recherche.

CIRANO is a private non-profit organization incorporated under the Québec Companies Act. Its infrastructure and research activities are funded through fees paid by member organizations, an infrastructure grant from the Ministère de l'Enseignement supérieur, de la Recherche, de la Science et de la Technologie, and grants and research mandates obtained by its research teams.

\section{Les partenaires du CIRANO}

\section{Partenaire majeur}

Ministère de l'Enseignement supérieur, de la Recherche, de la Science et de la Technologie

\section{Partenaires corporatifs}

Autorité des marchés financiers

Banque de développement du Canada

Banque du Canada

Banque Laurentienne du Canada

Banque Nationale du Canada

Banque Scotia

Bell Canada

BMO Groupe financier

Caisse de dépôt et placement du Québec

Fédération des caisses Desjardins du Québec

Financière Sun Life, Québec

\section{Gaz Métro}

Hydro-Québec

Industrie Canada

Investissements PSP

Ministère des Finances et de l'Économie

Power Corporation du Canada

Rio Tinto Alcan

Transat A.T.

Ville de Montréal

\section{Partenaires universitaires}

École Polytechnique de Montréal

École de technologie supérieure (ÉTS)

HEC Montréal

Institut national de la recherche scientifique (INRS)

McGill University

Université Concordia

Université de Montréal

Université de Sherbrooke

Université du Québec

Université du Québec à Montréal

Université Laval

Le CIRANO collabore avec de nombreux centres et chaires de recherche universitaires dont on peut consulter la liste sur son site web.

Les cahiers de la série scientifique (CS) visent à rendre accessibles des résultats de recherche effectuée au CIRANO afin de susciter échanges et commentaires. Ces cahiers sont écrits dans le style des publications scientifiques. Les idées et les opinions émises sont sous l'unique responsabilité des auteurs et ne représentent pas nécessairement les positions du CIRANO ou de ses partenaires.

This paper presents research carried out at CIRANO and aims at encouraging discussion and comment. The observations and viewpoints expressed are the sole responsibility of the authors. They do not necessarily represent positions of CIRANO or its partners. 


\title{
Identification-robust inference for endogeneity parameters in linear structural models
}

\author{
Firmin Doko Tchatoka ${ }^{+}$, Jean-Marie Dufour
}

\begin{abstract}
Résumé/abstract
We provide a generalization of the Anderson-Rubin (AR) procedure for inference on parameters which represent the dependence between possibly endogenous explanatory variables and disturbances in a linear structural equation (endogeneity parameters). We focus on second-order dependence and stress the distinction between regression and covariance endogeneity parameters. Such parameters have intrinsic interest (because they measure the effect of "common factors" which induce simultaneity) and play a central role in selecting an estimation method (because they determine "simultaneity biases" associated with least-square methods). We observe that endogeneity parameters may not be identifiable and we give the relevant identification conditions. These conditions entail a simple identification correspondence between regression endogeneity parameters and usual structural parameters, while the identification of covariance endogeneity parameters typically fails as soon as global identification fails. We develop identification-robust finite-sample tests for joint hypotheses involving structural and regression endogeneity parameters, as well as marginal hypotheses on regression endogeneity parameters. For Gaussian errors, we provide tests and confidence sets based on standard Fisher critical values. For a wide class of parametric non-Gaussian errors (possibly heavy-tailed), we also show that exact Monte Carlo procedures can be applied using the statistics considered. As a special case, this result also holds for usual AR-type tests on structural coefficients. For covariance endogeneity parameters, we supply an asymptotic (identificationrobust) distributional theory. Tests for partial exogeneity hypotheses (for individual potentially endogenous explanatory variables) are covered as special cases. The proposed tests are applied in three empirical examples: a model of the relation trade and economic growth; and two models of the returns to educations: the well-known study of Bound, Angrist and Balker (1995), and an alternative model considered by Card (1995).
\end{abstract}

Mots clés/keys words : Identification-robust confidence sets; endogeneity; AR-type statistic; projection-based techniques; partial exogeneity test.

Codes JEL : C3, C12, C15, C52.

\footnotetext{
* The authors thank Marine Carrasco, Jan Kiviet, Benoit Perron, Purevdorj Tuvaandorj, two anonymous referees, and the Editor Elie Tamer for several useful comments. This work was supported by the William Dow Chair in Political Economy (McGill University), the Bank of Canada (Research Fellowship), the Toulouse School of Economics (Pierre-de-Fermat Chair of excellence), the Universitad Carlos III de Madrid (Banco Santander de Madrid Chair of excellence), a Guggenheim Fellowship, a Konrad-Adenauer Fellowship (Alexander-vonHumboldt Foundation, Germany), the Canadian Network of Centres of Excellence [program on Mathematics of Information Technology and Complex Systems (MITACS)], the Natural Sciences and Engineering Research Council of Canada, the Social Sciences and Humanities Research Council of Canada, and the Fonds de recherche sur la société et la culture (Québec).

${ }^{\dagger}$ School of Economics and Finance, University of Tasmania, Private Bag 85, Hobart TAS 7001, Tel: +613 62267226, Fax:+61 36226 7587; e-mail: Firmin.dokotchatoka@utas.edu.au. Homepage:

http://www.fdokotchatoka.com

${ }^{\ddagger}$ William Dow Professor of Economics, McGill University, Centre interuniversitaire de recherche en analyse des organisations (CIRANO), and Centre interuniversitaire de recherche en économie quantitative (CIREQ). Mailing address: Department of Economics, McGill University, Leacock Building, Room 519, 855 Sherbrooke Street West, Montréal, Québec H3A 2T7, Canada. TEL: (1) 5143984400 ext. 09156; FAX: (1) 514398 4800; e-mail: jeanmarie.dufour@mcgill.ca.Web page: http://www.jeanmariedufour.com
} 


\section{Contents}

List of Definitions, Assumptions, Propositions and Theorems iii

1. Introduction 1

2. Framework: endogeneity parameters and their identification 3

2.1. Identification of endogeneity parameters . . . . . . . . . . . . . 4

2.2. Statistical problems . . . . . . . . . . . . . . . . . . . 9 9

3. Finite-sample inference for regression endogeneity parameters 10

3.1. AR-type tests for $\beta$ with possibly non-Gaussian errors . . . . . . . . . . . . 11

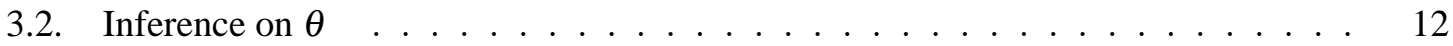

3.3. Joint inference on $\beta$ and regression endogeneity parameters $\ldots \ldots \ldots \ldots$

3.4. Confidence sets for regression endogeneity parameters . . . . . . . . . . . 14

3.5. Exact Monte Carlo identification-robust tests with non-Gaussian errors . . . . . 16

4. Asymptotic theory for inference on endogeneity parameters 17

$\begin{array}{llr}\text { 5. Empirical applications } & 18\end{array}$

5.1. Trade and growth . . . . . . . . . . . . . . . . . . . . 19

5.2. Angrist-Krueger model of education and earnings . . . . . . . . . . . . . . 19

5.3. Card model of education and earnings . . . . . . . . . . . . . . 20

$\begin{array}{llr}\text { 6. Conclusion } & 23\end{array}$

A. Proof 24 


\section{List of Tables}

1 Projection-based confidence sets for different parameters in earning equation . . . 20

2 Card model of education and earmings: projection-based confidence intervals for endogeneity parameters . . . . . . . . . . . . . . 22

\section{List of Figures}

\section{List of Definitions, Assumptions, Propositions and Theorems}

Assumption 2.0 : Structural homoskedasticity . . . . . . . . . . . . . 5

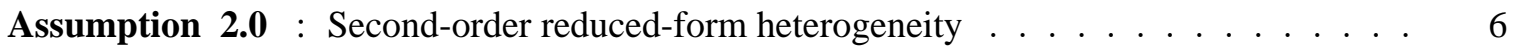

Assumption 2.0 : Reduced-form heterogeneity . . . . . . . . . . . . . . . 6

Lemma 2.1 : Identification of regression endogeneity parameters . . . . . . . . . . . 7

Proposition 2.2 : Equivalence between structural and reduced-form error decompositions 8

Assumption 3.0 : Conditional scale model for structural errors . . . . . . . . . . . . 10

Assumption 3.0 : Conditional scale model for orthogonalized structural errors . . . . . . 10

Proposition 3.1 : Null distribution of AR statistics under scale structural error model . 11 Proof of Proposition $3.1 \ldots \ldots \ldots \ldots$ 


\section{Introduction}

Instrumental variable (IV) regressions are typically motivated by the fact that "explanatory variables" may be correlated with the error term, so least-squares methods yield biased inconsistent estimators of model coefficients. Such IV parameter estimates can be interpreted as measures of the relationship between variables, once the "effect" of common "driving" (or "exogenous") variables has been eliminated. Even though coefficients estimated in this way may have interesting interpretations from the viewpoint of economic theory, inference on such "structural parameters" faces identification difficulties. Further, it is well known that IV estimators may be very imprecise, and inference procedures (such as tests and confidence sets) can be highly unreliable, especially when instruments are weakly associated with model variables (weak instruments). This has led to a large literature aimed at producing reliable inference in the presence of weak instruments; see the reviews of Stock, Wright and Yogo (2002) and Dufour (2003).

Research on weak instruments has focused on inference for the coefficients of endogenous variables in so-called "IV regressions". This leaves out the parameters which specifically determine simultaneity features, such as the covariances between endogenous explanatory variables and disturbances. These parameters can be of interest for several reasons. First, they provide direct measures of the importance of latent variables, which are typically unobserved and may simultaneously affect a number of observable endogenous variables. These latent variables are in a sense left out from structural equations, but they remain hidden in structural disturbances. For example, in a wide set of economic models, they may represent unobserved latent variables, such as "surprise variables" which play a role in models with expectations [see Barro (1977), Dufour and Jasiak (2001)]. Second, the simultaneity covariance (or regression) coefficients determine the estimation bias of leastsquares methods. Information on the size of such biases can be useful in interpreting least-squares estimates and related statistics. Third, information on the parameters of hidden variables (which induce simultaneity) may be important for selecting statistical procedures. Even if instruments are "strong", it is well known that IV estimators may be considerably less efficient than least-squares estimators; see Kiviet and Niemczyk (2007, 2012), Doko Tchatoka and Dufour (2011a), Kiviet and Pleus (2012) and Kiviet (2013). Indeed, this may be the case even when endogeneity is present. If a variable is not correlated (or only weakly correlated) with the error term, instrumenting it can lead to sizable efficiency losses in estimation. Assessing when and which variables should be instrumented is an important issue for the estimation of structural models.

We stress here the view that linear structural models (IV regressions) can be interpreted as regressions with missing regressors. If the latter were included, there would be no simultaneity bias, so no correction for simultaneity (such as IV methods) would be needed. This feature allows one to define a model transformation which maps a linear structural equation to a linear regression where all the explanatory variables are uncorrelated with the error term. We call this equation the orthogonalized structural equation, and we use it extensively. Interestingly, the latter is not a reduced-form equation. Instead, it involves the structural parameters of interest, but also includes endogeneity parameters which are "hidden" in the original structural equation.

The problem stems from the fact that the missing regressors are unobserved. Despite this difficulty, we show that procedures similar to the one proposed by Anderson and Rubin (1949, AR) 
can be applied to the orthogonalized equation. This allows one to make inference jointly on both the parameters of the original structural equation and endogeneity parameters. Two types of endogeneity parameters are considered: regression endogeneity parameters and covariance endogeneity parameters. Under standard conditions, where instruments are strictly exogenous and errors are Gaussian, the tests and confidence sets derived in this way are exact. The proposed methods do not require identification assumptions, so they can be characterized as identification-robust. For more general inference on transformations of the parameters in the orthogonalized structural equation, we propose projection methods, for such techniques allow for a simple finite-sample distributional theory and preserve robustness to identification assumptions.

To be more specific, we consider a model of the form

$$
y=Y \beta+X_{1} \gamma+u
$$

where $y$ is an observed dependent variable, $Y$ is a matrix of observed (possibly) endogenous regressors, and $X_{1}$ is a matrix of exogenous variables. We observe that AR-type procedures may be applied to test hypotheses on the transformed parameter $\theta=\beta+a$, where $a$ represents regression coefficients of $u$ on the reduced-form errors of $Y$ (regression endogeneity parameters). Identification-robust inference for $a$ itself is then derived by exploiting the possibility of making identification-robust inference on $\beta$. Then, inference on covariances (say $\sigma_{V u}$ ) between $u$ and $Y$ (covariance endogeneity parameters) can be derived by considering linear transformations of $a$.

We stress that regression and covariance endogeneity parameters - though theoretically related - play distinct but complementary roles: regression endogeneity parameters represent the effect of reduced-form innovations on $y$, while covariance endogeneity parameters determine the need to instrument different variables in $Y$. When $\sigma_{V u}=0, Y$ can be treated as exogenous (so IV estimation is not warranted). So-called exogeneity tests typically test the hypothesis $\sigma_{V u}=0$. It is easy to see that $\sigma_{V u}=0$ if and only if $a=0$ (provided the covariance matrix between reduced-form errors is nonsingular), but the relationship is more complex in other cases.

In this paper, we emphasize cases where $a \neq 0$. We first study formally the identification of endogeneity parameters. We establish a simple identification correspondence between the components of $\beta$ and $a$ : each component of $a$ is identifiable if and only if the corresponding component of $\beta$ is identifiable. In contrast, this does not hold in general for the covariances $\sigma_{V u}$ : as soon as one element of $\beta$ is not identifiable, all components of $\sigma_{V u}$ typically fail to be identifiable. In this sense, $\sigma_{V u}$ is more difficult to interpret than $a$. Due to the failure of the exogeneity hypothesis, the distributions of the test statistics are much more complex. It is relatively easy to produce finite-sample inference for $a$, but not for $\sigma_{V u}$. So, for $\sigma_{V u}$, we propose asymptotic tests and confidence sets. It is important to note that stronger assumptions are needed for making inference on $\sigma_{V u}$ (as opposed to $a$ ). Indeed, we describe general distributional setups where $\sigma_{V u}$ may not be well-defined [due to heterogeneity in the model for $Y$, or the non-existence of moments], while $a$ remains well-defined and statistically meaningful. In such cases, inference on $a$ is feasible, while inference on $\sigma_{V u}$ may not be (even when all parameters in the structural equation of interest are identifiable).

By allowing $a \neq 0$ (or $\sigma_{V u} \neq 0$ ), we extend earlier results on exogeneity tests, which focus on the null hypothesis $H_{a}: a=0$. The literature on this topic is considerable; see, for example, Durbin (1954), Wu (1973, 1974, 1983a, 1983b), Revankar and Hartley (1973), Farebrother 
(1976), Hausman (1978), Revankar (1978), Dufour (1979, 1987), Hausman and Taylor (1981), Hwang (1980, 1985), Kariya and Hodoshima (1980), Hausman and Taylor (1981), Spencer and Berk (1981), Nakamura and Nakamura (1981, 1985), Engle (1982), Holly (1982, 1983b, 1983a), Smith (1983, 1984, 1985), Ruud (1984, 2000), Davidson and Mackinnon (1985, 1985, 1989, 1990, 1993), Newey (1985a, 1985b), Thurman (1986), Smith and Pesaran (1990), Meepagala (1992), Wong (1996), Ahn (1997), Staiger and Stock (1997), , Baum, Schaffer and Stillman (2003), Hahn, Ham and Moon (2010), Chmelarova and Hill (2010), Jeong and Yoon (2010), and Kiviet and Pleus (2012).

By contrast, we consider here the problem of testing any value of $a$ (or $\sigma_{V u}$ ) and build confidence sets for these parameters. By allowing for weak instruments, we extend the results in Dufour (1979, 1987) where Wald-type tests and confidence sets are proposed for inference on $a$ and $\sigma_{V u}$, under assumptions which exclude weak instruments. Finally, by considering inference on $a$ and $\sigma_{V u}$, we extend a procedure proposed in Dufour and Jasiak (2001) for inference on the aggregate parameter $\theta=\beta+a$ (but not $a$ or $\sigma_{V u}$ ) in the context of a different model.

On exploiting results from Dufour and Taamouti (2005, 2007), we supply analytical forms for the proposed confidence sets, and we give the necessary and sufficient conditions under which they are bounded. These results can be used to assess partial exogeneity hypotheses even when identification is deficient or weak. In order to allow for alternative assumptions on error distributions, we show that the proposed AR-type statistics are pivotal as long as the errors follow a completely specified distribution (up to an unknown scale parameter), which may be non-Gaussian. Due to this invariance property, exact Monte Carlo tests can be performed without a Gaussian assumption [as described in Dufour (2006)]. In particular, we show this is feasible under general assumptions which allow considerable heterogeneity in the reduced-form model for $Y$, even a completely unspecified model for $Y$. On allowing for more general error distributions and weakly exogenous instruments (along with standard high-level asymptotic assumptions), we also show that the proposed procedures remain asymptotically valid and identification-robust.

Finally, we apply the proposed methods to three empirical examples: a model of the relation between trade and economic growth, previously studied in Frankel and Romer (1999) and Dufour and Taamouti (2007); and two models of the returns to educations, the well-known study of Bound, Jaeger and Baker (1995), and an alternative model considered by Card (1995) and Kleibergen (2004, Table 2, p. 421).

The paper is organized as follows. Section 2 describes the model and the identification conditions for endogeneity parameters. Section 3 presents the finite-sample theory for inference on regression endogeneity parameters. Section 4 discusses asymptotic theory and inference for covariance endogeneity parameters. Section 5 illustrates the theoretical results through three empirical applications: a model of the relationship between trade and growth, and two models of returns to schooling. We conclude in Section 6. Proofs are presented in appendix.

\section{Framework: endogeneity parameters and their identification}

We consider a standard linear structural equation of the form: 


$$
y=Y \beta+X_{1} \gamma+u
$$

where $y=\left[y_{1}, \ldots, y_{T}\right]$ is a $T \times 1$ vector of observations on a dependent variable, $Y=\left[Y_{1}, \ldots, Y_{T}\right]^{\prime}$ is a $T \times G$ matrix of observations on (possibly) endogenous explanatory variables $(G \geq 1), X_{1}$ is a $T \times k_{1}$ full-column-rank matrix of strictly exogenous variables, $u=\left[u_{1}, \ldots, u_{T}\right]^{\prime}$ is a vector of structural disturbances, $\beta$ and $\gamma$ are $G \times 1$ and $k_{1} \times 1$ unknown coefficient vectors. Further, $Y$ satisfies the model:

$$
Y=X \Pi+V=X_{1} \Pi_{1}+X_{2} \Pi_{2}+V
$$

where $X_{2}$ is a $T \times k_{2}$ matrix of observations on exogenous variables (instruments), $X=\left[X_{1}, X_{2}\right]=$ $\left[X_{\bullet 1}, \ldots, X_{\bullet T}\right]^{\prime}$ has full-column rank $k=k_{1}+k_{2}, \Pi_{1}$ and $\Pi_{2}$ are $k_{1} \times G$ and $k_{2} \times G$ coefficient matrices, $\Pi=\left[\Pi_{1}, \Pi_{2}\right]$, and $V=\left[V_{1}, \ldots, V_{T}\right]^{\prime}$ is a $T \times G$ matrix of reduced-form disturbances. Equation (2.1) is the "structural equation" of interest, while (2.2) represents the "reduced form" for $Y$. On substituting (2.2) into (2.1), we get the reduced form for $y$ :

$$
y=X_{1} \pi_{1}+X_{2} \pi_{2}+v
$$

where $\pi_{1}=\gamma+\Pi_{1} \beta, \pi_{2}=\Pi_{2} \beta$, and $v=V \beta+u=\left[v_{1}, \ldots, v_{T}\right]^{\prime}$.

When the errors $u$ and $V$ have finite means (although this assumption could easily be replaced by another "location assumption", such as zero medians), the usual necessary and sufficient condition for identification of $\beta$ and $\gamma$ (from the first moments of $y$ and $Y$ ) in (2.1) - (2.2) is:

$$
\operatorname{rank}\left(\Pi_{2}\right)=G
$$

If $\Pi_{2}=0$, the instruments $X_{2}$ are irrelevant, and $\beta$ is completely unidentified. If $1 \leq \operatorname{rank}\left(\Pi_{2}\right)<G$, $\beta$ is not identifiable, but some linear combinations of the elements of $\beta$ are identifiable [see Dufour and Hsiao (2008)]. If $\Pi_{2}$ is close not to have full rank [e.g., if some eigenvalues of $\Pi_{2}^{\prime} \Pi_{2}$ are close to zero], some linear combinations of $\beta$ are ill-determined by the data, a situation often called "weak identification" [see Dufour (2003)].

Throughout this paper, $I_{m}$ is the identity matrix of order $m$. For any full-column-rank $T \times m$ matrix $A, P(A)=A\left(A^{\prime} A\right)^{-1} A^{\prime}, M(A)=I_{T}-P(A)$, vec $(A)$ is the $(T m) \times 1$ column vectorization of $A$, and $\|A\|=\left[\operatorname{tr}\left(A^{\prime} A\right)\right]^{\frac{1}{2}}$ the matrix Euclidian norm. For $A$ square, $A>0$ means $A$ is positive definite (p.d.), and $A \geq 0$ means $A$ is positive semidefinite (p.s.d.). " $\stackrel{p}{\rightarrow}$ " stands for convergence in probability, and “ $\stackrel{L}{\rightarrow}$ ” for convergence in distribution.

\subsection{Identification of endogeneity parameters}

We now wish to represent the fact that $u$ and $V$ can be correlated, allowing for the possibility of identification failure. It is important to note that the "structural error" $u_{t}$ may not be uniquely determined by the data when $\beta$ and $\gamma$ are not identified. For this, it will be useful to consider two alternative setups for the disturbance distribution: (A) in the first one, the disturbance vectors $\left(u_{t}, V_{t}^{\prime}\right)^{\prime}$ have common finite second moments (structural homoskedasticity); (B) in the second one, we allow for a large amount of heterogeneity in the distributions of reduced-form errors (reducedform heterogeneity). The second setup is more appropriate for practical work, and we wish to go as 
far as possible in that direction. But it will be illuminating to first consider setup A.

Assumption A Structural homoskedasticity. The vectors $U_{t}=\left(u_{t}, V_{t}^{\prime}\right)^{\prime}, t=1, \ldots, T$, all have finite second moments with mean zero and the same covariance matrix

$$
\Sigma_{U}=\mathbb{E}\left[U_{t} U_{t}^{\prime}\right]=\left[\begin{array}{cc}
\sigma_{u}^{2} & \sigma_{V u}^{\prime} \\
\sigma_{V u} & \Sigma_{V}
\end{array}\right], \text { where } \Sigma_{V}=\mathbb{E}\left[V_{t} V_{t}^{\prime}\right] \text { is nonsingular. }
$$

Under the above assumption, we have:

$$
\sigma_{V v}=\mathbb{E}\left[V_{t} v_{t}\right]=\mathbb{E}\left[V_{t}\left(V_{t}^{\prime} \beta+u_{t}\right]=\Sigma_{V} \beta+\sigma_{V u}, \sigma_{v}^{2}=\sigma_{u}^{2}+\beta^{\prime} \Sigma_{V} \beta+2 \beta^{\prime} \sigma_{V u} .\right.
$$

The covariance vector $\sigma_{V u}$ indicates which variables in $Y$ are "correlated" with $u_{t}$, so it is a basic determinant of the level of "endogeneity" of these variables. Note, however, that $\sigma_{V u}$ is not identifiable when $\beta$ is not (for then the "structural error" $u_{t}$ is not uniquely determined by the data).

In this context, it will be illuminating to look at the following two regressions: (1) the linear regression of $u_{t}$ on $V_{t}$,

$$
u_{t}=V_{t}^{\prime} a+e_{t}, t=1, \ldots, T,
$$

where $a=\Sigma_{V}^{-1} \sigma_{V u}$ and $\mathbb{E}\left[V_{t} e_{t}\right]=0$ for all $t$; and (2) the linear regression of $v_{t}$ on $V_{t}$,

$$
v_{t}=V_{t}^{\prime} \theta+\eta_{t}, t=1, \ldots, T
$$

where $\theta=\Sigma_{V}^{-1} \sigma_{V v}$ and $\mathbb{E}\left[V_{t} \eta_{t}\right]=0$ for all $t$. It is easy to see that

$$
\sigma_{V u}=\Sigma_{V} a, \quad \sigma_{u}^{2}=\sigma_{e}^{2}+a^{\prime} \Sigma_{V} a=\sigma_{e}^{2}+\sigma_{V u}^{\prime} \Sigma_{V}^{-1} \sigma_{V u},
$$

where $\mathbb{E}\left[e_{t}^{2}\right]=\sigma_{e}^{2}$ for all $t$. This entails that: $a=0$ if and only if $\sigma_{V u}=0$, so the exogeneity of $Y$ can be assessed by testing whether $a=0$. There is however no simple match between the components of $a$ and $\sigma_{V u}$ (unless $\Sigma_{V}$ is a diagonal matrix). For example, if $a=\left(a_{1}^{\prime}, a_{2}^{\prime}\right)^{\prime}$ and $\sigma_{V u}=\left(\sigma_{V u 1}^{\prime}, \sigma_{V u 2}^{\prime}\right)^{\prime}$ where $a_{1}$ and $\sigma_{V u 1}$ have dimension $G_{1}<G, a_{1}=0$ is not equivalent to $\sigma_{V u 1}=0$. We call $a$ the "regression endogeneity parameter", and $\sigma_{V u}$ the "covariance endogeneity parameter".

As long as the identification condition (2.4) holds, both $\sigma_{V u}$ and $a$ are identifiable. This is not the case, however, when (2.4) does not hold. By contrast, the regression coefficient $\theta$ is always identifiable, because it is uniquely determined by the second moments of reduced-form errors. It is then useful to observe the following identity:

$$
\theta=\Sigma_{V}^{-1} \sigma_{V v}=\Sigma_{V}^{-1}\left(\Sigma_{V} \beta+\sigma_{V u}\right)=\beta+a .
$$

In other words, the sum $\beta+a$ is equal to the regression coefficient of $v_{t}$ on $V_{t}$. Even though $\beta$ and $a$ may not be identifiable, the sum $\beta+a$ is identifiable (from the first and second moments of $v$ and $V$ ). Further, for any fixed $G \times 1$ vector $w, w^{\prime} \theta$ is identifiable, so the identities $w^{\prime} a=w^{\prime} \theta-w^{\prime} \beta$ and $\sigma_{V u}=$ $\Sigma_{V} a$ along with the invertibility of $\Sigma_{V}$ entail the following equivalences:

$$
\beta \text { is identifiable } \Leftrightarrow a \text { is identifiable } \Leftrightarrow \sigma_{V u} \text { is identifiable ; }
$$




$$
w^{\prime} \beta \text { is identifiable } \Leftrightarrow w^{\prime} a \text { is identifiable } \Leftrightarrow w^{\prime} \Sigma_{V}^{-1} \sigma_{V u} \text { is identifiable . }
$$

In particular, (2.12) entails a simple identification correspondence between the components of $\beta$ and $a$ : for each $1 \leq i \leq G, a_{i}$ is identifiable $\Leftrightarrow \beta_{i}$ is identifiable. In other words, the identification conditions for $\beta$ and $a$ are identical. In contrast, the equivalences $\left[w^{\prime} \sigma_{V u}\right.$ is identifiable $\Leftrightarrow w^{\prime} \beta$ is identifiable] and [ $\sigma_{V u i}$ is identifiable $\Leftrightarrow \beta_{i}$ is identifiable] do not hold in general: as soon as one element of $\beta$ is not identifiable, all components of $\sigma_{V u}$ typically fail to be identifiable. In this sense, $\sigma_{V u}$ is more difficult to interpret than $a$.

Setup A requires that the reduced-form disturbances $V_{t}, t=1, \ldots, T$, have identical second moments. In many practical situations, this may not be appropriate, especially in a limited-information analysis which focuses on the structural equation of interest (2.1), rather than the marginal distribution of the explanatory variables $Y$. To allow for more heterogeneity among the observations in $Y$, we consider the following alternative assumptions (where $X_{\bullet}$ is the $t$-th row of $X$ ).

Assumption B SECOND-ORDER REDUCED-FORM HETEROGENEITY. For some fixed vector a in $\mathbb{R}^{G}$, we have:

$$
u=V a+e
$$

where $e, V$ and $X$ have finite second moments, $\mathbb{E}[e]=0$, and $e$ is uncorrelated with $V$ and $X$.

Assumption C Reduced-Form Heterogeneity. Equation (2.13) holds with $\mathbb{E}\left[e_{t} \mid V_{t}, X_{\bullet}\right]=$ $0, t=1, \ldots, T$.

Assumptions B and C allow substantial heterogeneity in the distribution of the disturbances $V_{t}, t=1, \ldots, T$. The latter need not be identically distributed or independent. Assumption B maintains the existence of second moments [even though the covariance matrices $\mathbb{E}\left(V_{t} V_{t}^{\prime}\right)$ may vary with $t$ ] and defines $e$ through a zero mean and orthogonality with $V$ and $X$. Assumption $C$ replaces this condition by a zero conditional mean; no further restriction on $V$ is imposed. The existence of moments for $V_{t}$ and $X_{\bullet t}$ is not required. An important case where Assumption B holds is the one where $V$ and $e$ are independent (strong linear structural decomposition). Given (2.1), the three conditions $\mathbb{E}\left[e_{t} \mid V_{t}, X_{\bullet t}\right]=0, \mathbb{E}\left[e_{t} \mid Y_{t}, X_{\bullet t}\right]=0$ and $\mathbb{E}\left[e_{t} \mid Y_{t}, V_{t}, X_{\bullet t}\right]=0$ are equivalent. In such cases, $\sigma_{V u}$ may not be well-defined [due to heterogeneity in the model for $Y$, or the non-existence of moments], but $a$ remains statistically meaningful.

In view of the decomposition (2.13), equation (2.1) can be viewed as a regression model with missing regressors. On substituting (2.13) into (2.1), we get:

$$
y=Y \beta+X_{1} \gamma+V a+e
$$

where $e$ is uncorrelated with all the regressors. Because of this property, we call (2.14) the orthogonalized structural equation associated with (2.2), and $e$ the orthogonalized structural disturbance vector. ${ }^{1}$ In this equation, the original structural parameters $(\beta$ and $\gamma)$ can be interpreted as regres-

\footnotetext{
${ }^{1}$ The form (2.14) was orignally proposed by Revankar and Hartley (1973) for the purpose of testing complete exogeneity $(a=0)$. As pointed out in Dufour $(1979,1987)$, the disributional theory is substantially simpler in that case and does not allow one to test more general restrictions on $a$ (because the covariance matrix is modified).
} 
sion coefficients, along with the regression endogeneity parameter $a$. We see that $a$ represents the effect of the latent variable $V$. Even though (2.14) is a regression equation $\left[\left(Y, X_{1}, V\right)\right.$ is orthogonal to the disturbance $e$ ], it is quite distinct from the reduced-form equation (2.3) for $y$.

The orthogonalized structural equation is quite helpful for interpreting model coefficients. A structural model of the form (2.1) - (2.2) often represents a causal structure to explain $y$. The endogenous variables $(y$ and $Y$ ) are determined by two types of inputs: observable exogenous variables $\left(X_{1}\right.$ and $\left.X_{2}\right)$ and unobserved variables $(V$ and $e)$. $X_{1}$ has both a direct effect $\left(X_{1} \gamma\right)$ on $y$ and an indirect effect $\left(X_{1} \Pi_{1} \beta\right.$ through $\left.Y\right)$, while $X_{2}$ only has an indirect effect $\left(X_{2} \Pi_{2} \beta\right)$. Similarly, $V$ represents unobserved variables (e.g., shocks, latent variables, expectation errors) which have both a direct effect $(V a)$ and an indirect effect $(V \beta)$, while $e$ represents idiosyncratic shocks to $y$ which are orthogonal to $Y$. Finally, we may interpret the sum $V \beta+V a=V(\beta+a)$ as the net final effect (both direct and indirect) of $V$ on $y$. In the context of a causal interpretation, the coefficient vectors $\beta, a$ and $\beta+a$ have useful distinct interpretations: $\beta$ represents the impact of $Y$ [in particular, its systematic component $E(Y)=X_{1} \Pi_{1}+X_{2} \Pi_{2}$ ] on $y, a$ the direct effect of the latent variable $V$ on $y$, and $\beta+a$ the total effect of $V$ on $y$. Statistical inference on each one of the coefficients has its own independent interest.

The identification of $a$ can be studied through the orthogonalized structural equation. By (2.2),

$$
y=Y \theta+X_{1} \pi_{1}^{*}+X_{2} \pi_{2}^{*}+e
$$

where $\theta=\beta+a, \pi_{1}^{*}=\gamma-\Pi_{1} a, \pi_{2}^{*}=-\Pi_{2} a$, and $e$ is uncorrelated with all the regressors $\left(Y, X_{1}\right.$ and $X_{2}$ ). Equation (2.15) is a regression equation obtained by adding $X_{2}$ to the original structural equation or, equivalently, by adding $Y$ to the reduced form (2.3) for $y$. We call (2.15) the extended reduced form associated with (2.2). As soon as the matrix $Z=\left[Y, X_{1}, X_{2}\right]$ has full-column rank with probability one [almost surely (a.s.)], the parameters of equation (2.15) are identifiable (a.s.), because they are uniquely determined by the linear projections of $y_{t}$ on $Y_{t}$ and $X_{\bullet t}$ for $t=1, \ldots, T$ [under Assumption B] or by the corresponding conditional means [under assumption $\mathrm{C}$ ]. This is the case in particular for $\theta=\beta+a$ (with probability one) when $Z$ has full-column rank with probability one. This rank condition holds in particular when the matrix $V$ has full column rank (a.s., conditional on $X), e . g$. if its distribution is absolutely continuous. This entails again that $a$ is identifiable if and only if $\beta$ is identifiable, and similarly between $w^{\prime} a$ and $w^{\prime} \beta$ for any $w \in \mathbb{R}^{G}$. This establishes the following identification result for $a$, where "identification" refers to the conditional distributions of $y_{t}$ given $Y_{t}$ and $X_{\bullet}, t=1, \ldots, T$.

Proposition 2.1 IDENTIFICATION OF REGRESSION ENDOGENEITY PARAMETERS. Under the model given by (2.2), (2.3) and Assumption B or $C$, suppose the matrix $\left[Y, X_{1}, X_{2}\right]$ has full column rank. Then $a+\beta$ is identifiable, and the following two equivalences hold:

$$
\begin{gathered}
\text { a is identifiable } \Leftrightarrow \beta \text { is identifiable; } \\
\text { for any } w \in \mathbb{R}^{G}, w^{\prime} \text { a is identifiable } \Leftrightarrow w^{\prime} \beta \text { is identifiable. }
\end{gathered}
$$

The decomposition assumption (2.13) can also be formulated in terms of the reduced-form disturbance $v$ [as in (2.8)] rather than the structural disturbance $u$ : 


$$
v=V \bar{\theta}+\eta
$$

for some fixed vector $\bar{\theta}$ in $\mathbb{R}^{G}$, where each element of $\eta$ has mean zero and is uncorrelated with $V$ and $X$, again without any other assumption on the distribution of $V$. This means that the linear regressions $v_{t}=V_{t}^{\prime} \bar{\theta}+\eta_{t}, t, \ldots, T$, can all be written in terms of the same coefficient vector $\bar{\theta}$. The latter is uniquely determined (identifiable) as soon as the matrix $V$ has full column rank (with probability one), so the identification of $\beta$ is irrelevant. Even though conditions (2.13) and (2.18) are quite different (because the dependent variable is not the same), they are equivalent in the context of the model we study here. This can be seen by rewriting the reduced form (2.3) as follows:

$$
\begin{aligned}
y & =X_{1} \pi_{1}+X_{2} \pi_{2}+v=X_{1}\left(\gamma+\Pi_{1} \beta\right)+X_{2}\left(\Pi_{2} \beta\right)+V \bar{\theta}+\eta \\
& =\left(X_{1} \Pi_{1}+X_{2} \Pi_{2}\right) \beta+X_{1} \gamma+V \bar{\theta}+\eta=Y \beta+X_{1} \gamma+V(\bar{\theta}-\beta)+\eta .
\end{aligned}
$$

By matching the latter equation with the structural form (2.1), we get

$$
u=V(\bar{\theta}-\beta)+\eta
$$

provided $\left[Y, X_{1}\right]$ has full-column rank. Since $\eta$ and $V$ are uncorrelated, this entails that (2.13) holds with $a=\bar{\theta}-\beta$ and $e=\eta$. Conversely, under Assumption $\mathrm{B}$, we have from the reduced form (2.3):

$$
v=V \beta+u=V(\beta+a)+e
$$

which is equivalent to (2.18) with $\bar{\theta}=\beta+a=\theta$ and $\eta=e$. We can thus state the following proposition.

Proposition 2.2 EquivalenCE BetWeEn STRUCTURAL AND REDUCED-FORM ERROR DECOMPOSITIONS. Under the assumptions (2.2) and (2.3), suppose the matrix $\left[Y, X_{1}, X_{2}\right]$ has full column rank with probability one. Then the assumptions (2.13) and (2.18) are equivalent with $\bar{\theta}=\beta+a$ and $\eta=e$.

The identity $\eta=e$ entails that the residual vector from the regression of $u$ on $V$ is uniquely determined (identifiable) even if $u$ itself may not be. The orthogonalized structural equation (2.14) may thus be rewritten as

$$
y=Y \beta+X_{1} \gamma+V(\bar{\theta}-\beta)+\eta=(X \Pi) \beta+X_{1} \gamma+V \bar{\theta}+\eta
$$

where $\bar{\theta}$ is a regression vector between two reduced-form disturbances $(v$ on $V)$ and $\eta$ the corresponding error. This shows clearly that different regression endogeneity parameters $a=\bar{\theta}-\beta$ are obtained by "sweeping" $\beta$ over its identification set.

Under Assumption B, covariance endogeneity parameters may depend on $t$. Indeed, it is easy to see that $\mathbb{E}\left[V_{t} u_{t}\right]=\mathbb{E}\left[V_{t} V_{t}^{\prime}\right] a \equiv \sigma_{V u t}$, which may depend on $t$ if $\mathbb{E}\left[V_{t} V_{t}^{\prime}\right]$ does. However, identification of the parameters $\sigma_{V u t}$ remains determined by the identification of $a$, whenever the reducedform covariance (which are parameters of reduced forms) are identifiable. Inference on covariance endogeneity parameters requires additional assumptions. In sections 3 and 4, we will see that finitesample inference methods can be derived for regression endogeneity parameters under the relatively 
"weak" Assumption B, while only asymptotically justified methods will be proposed for covariance endogeneity parameters. For covariances, we will focus on the case where $\sigma_{V u t}$ is constant.

\subsection{Statistical problems}

In this paper, we consider the problem of testing hypotheses and building confidence sets for regression endogeneity parameters $(a)$ and covariance endogeneity parameters $\left(\sigma_{V u}\right)$, allowing for the possibility of identification failure (or weak identification). We develop inference procedures for the full vectors $a$ and $\sigma_{V u}$, as well as linear transformations of these parameters $w^{\prime} a$ and $w^{\prime} \sigma_{V u}$. In view of the identification difficulties present here, we emphasize methods for which a finite-sample distributional theory is possible [see Dufour $(1997,2003)$ ], at least partially.

In line with the above discussion of the identification of endogeneity parameters, we observe that inference on $a$ can be tackled more easily than inference on $\sigma_{V u}$, so we study this problem first. The problem of testing hypotheses of the form $H_{a}\left(a_{0}\right): a=a_{0}$ can be viewed as an extension of the classical Anderson and Rubin $(1949, \mathrm{AR})$ problem on testing $H_{\beta}\left(\beta_{0}\right): \beta=\beta_{0}$. There is, however, an additional complication: the variable $V$ is not observable. For this reason, substantial adjustments are required. To achieve our purpose, we propose a strategy that builds on two-stage confidence procedures [Dufour (1990)], projection methods [Dufour (1990, 1987), Abdelkhalek and Dufour (1998), Dufour and Jasiak (2001), Dufour and Taamouti (2005)], and Monte Carlo tests [Dufour (2006)].

Specifically, in order to build a confidence set with level $1-\alpha$ for $a$, choose $\alpha_{1}$ and $\alpha_{2}$ such that $0<\alpha=\alpha_{1}+\alpha_{2}<1,0<\alpha_{1}<1$ and $0<\alpha_{2}<1$. We can then proceed as follows:

(1) we build an identification-robust confidence set with level $1-\alpha_{1}$ for $\beta$; several methods are available to do this; in view of the existence of a finite-sample distributional theory (as well as computational simplicity), we focus on the Anderson and Rubin (1949, AR) approach; but alternative procedures could be exploited for that purpose, ${ }^{2}$

(2) we build an identification-robust confidence set for the sum $\theta=\beta+a$, which happens to be an identifiable parameter; we show this can be done easily though simple regression methods;

(3) the confidence sets for $\beta$ and $\theta$ are combined to obtain a simultaneous confidence set for the stacked parameter vector $\varphi=\left(\beta^{\prime}, \theta^{\prime}\right)^{\prime}$; by the Boole-Bonferroni inequality, this yields a confidence set for $\varphi$ with level $1-\alpha$ (at least), as in Dufour (1990);

(4) confidence sets for $a=\theta-\beta$ and any linear transformation $w^{\prime} a$ may then be derived by projection; these confidence sets have level $1-\alpha$;

(5) confidence sets for $\sigma_{V u}$ and $w^{\prime} \sigma_{V u}$ can finally be built using the relationship $\sigma_{V u}=\Sigma_{V} a$.

For inference on $a$, we develop a finite-sample approach which remains valid irrespective of assumptions on the distribution of $V$. In addition, we observe that the test statistics used for inference on $\beta$ [the AR-type statistic] and $\theta$ enjoy invariance properties which allow the application of Monte Carlo test methods: as long as the distribution of the errors $u$ is specified up to an unknown scale parameter, exact tests can be performed on $\beta$ and $\theta$ through a small number of Monte Carlo simulations [see Dufour (2006)]. For inference on both regression and covariance endogeneity parameters

\footnotetext{
${ }^{2}$ Such procedures include, for example, the methods proposed by Kleibergen (2002) or Moreira (2003). No finitesample distributional theory is, however, available for these methods. Further, these are not robust to missing instruments; see Dufour (2003) and Dufour and Taamouti (2007).
} 
( $a$ and $\sigma_{V u}$ ), we also provide a large-sample distributional theory based on standard asymptotic assumptions which relax various restrictions used in the finite-sample theory. All proposed methods do not make identification assumptions on $\beta$, either in finite samples or asymptotically.

\section{Finite-sample inference for regression endogeneity parameters}

In this section, we study the problem of building identification-robust tests and confidence sets for the regression endogeneity parameter $a$ from a finite-sample viewpoint. Along with (2.1) - (2.2), we suppose that Assumption B holds under (at least) one of the following conditions on model disturbances.

Assumption 3.1 COnditional SCALE MOdel FOR STRUCTURAL ERRORS. $u=\sigma(X) v$, where $\sigma(X)$ is a (possibly random) function of $X$ such that $\mathbb{P}[\sigma(X) \neq 0 \mid X]=1$, and the conditional distribution of $v$ given $X$ is completely specified.

Assumption 3.2 CONDITIONAL SCALE MOdEL FOR ORTHOGONALIZED STRUCTURAL ERRORS. $e=\sigma_{1}(X) \varepsilon$, where $\sigma_{1}(X)$ is a (possibly random) function of $X$ such that $\mathbb{P}\left[\sigma_{1}(X) \neq 0 \mid X\right]=1$, and the conditional distribution of $\varepsilon$ given $X$ is completely specified.

Assumption 3.1 means the distribution of $u$ given $X$ only depends on $X$ and a (typically unknown) scale factor $\sigma(X)$. The scale factor can also be random, so we can have $\sigma(X)=\bar{\sigma}(X, v)$. Of course, this holds when ever $u=\sigma v$, where $\sigma$ is an unknown positive constant and $v$ is independent of $X$ with a completely specified distribution. In this context, the standard Gaussian assumption is obtained by taking: $v \sim \mathrm{N}\left[0, I_{T}\right]$. But non-Gaussian distributions are covered, including heavy-tailed distributions which may lack moments (such as the Cauchy distribution). Similarly, Assumption 3.2 means the distribution of $e$ given $X$ only depends on $X$ and a (typically unknown, possibly random) scale factor $\sigma_{1}(X)$, so again a standard Gaussian model is obtained by assuming that $\sigma_{1}(X)$ is fixed (given $X$ ) and $\varepsilon \sim \mathrm{N}\left[0, I_{T}\right]$. In general, assumptions 3.1 and 3.2 do not entail each other. However, it is easy to see that both hold when the vectors $\left[u_{t}, V_{t}^{\prime}\right]^{\prime}, t, \ldots, T$, are i.i.d. (given $X$ ) with finite second moments and the decomposition specified by Assumption B holds. This will be the case a fortiori if the vectors $\left[u_{t}, V_{t}^{\prime}\right]^{\prime}, t, \ldots, T$, are i.i.d. multinormal (given $X$ ).

We will study in turn the following problems:

1. test and build confidence sets for $\beta$;

2. test and build confidence sets for $\theta=\beta+a$;

3. test and build confidence sets for $a$;

4. test and build confidence sets for scalar linear transformations $w^{\prime} a$. 


\subsection{AR-type tests for $\beta$ with possibly non-Gaussian errors}

Since this will be a basic building block for inference on endogeneity parameters, we consider first the problem of testing the hypothesis

$$
H_{\beta}\left(\beta_{0}\right): \beta=\beta_{0}
$$

where $\beta_{0}$ is any given possible value of $\beta$. Several methods have been proposed for that purpose. However, since we wish to use an identification-robust procedure for which a finite-sample theory can easily be obtained and does not require assumptions on the distribution of $Y$, we focus on the Anderson and Rubin (1949, AR) procedure. So we consider the transformed equation:

$$
y-Y \beta_{0}=X_{1} \pi_{1}^{0}+X_{2} \pi_{2}^{0}+v^{0}
$$

where $\pi_{1}^{0}=\gamma+\Pi_{1}\left(\beta-\beta_{0}\right), \pi_{2}^{0}=\Pi_{2}\left(\beta-\beta_{0}\right)$ and $v^{0}=u+V\left(\beta-\beta_{0}\right)$. Since $\pi_{2}^{0}=0$ under $H_{\beta}\left(\beta_{0}\right)$, it is natural to consider the corresponding $F$-statistic in order to test $H_{\beta}\left(\beta_{0}\right)$ :

$$
A R\left(\beta_{0}\right)=\frac{\left(y-Y \beta_{0}\right)^{\prime}\left(M_{1}-M\right)\left(y-Y \beta_{0}\right) / k_{2}}{\left(y-Y \beta_{0}\right)^{\prime} M\left(y-Y \beta_{0}\right) /(T-k)}
$$

where $M_{1} \equiv M\left(X_{1}\right)$ and $M \equiv M(X)$. Under the usual assumption where $u \sim \mathrm{N}\left[0, \sigma^{2} I_{T}\right]$ independently of $X$, the conditional distribution of $A R\left(\beta_{0}\right)$ under $H_{\beta}\left(\beta_{0}\right)$ is $F\left(k_{2}, T-k\right)$. In the following proposition, we characterize by invariance the distribution of $A R\left(\beta_{0}\right)$ under the general Assumption 3.1 .

Proposition 3.1 Null Distribution of AR Statistics Under SCAlE STRUCTURAL ERROR MODEL. Suppose the assumptions (2.1), (2.2) and 3.1 hold. If $\beta=\beta_{0}$, we have:

$$
A R\left(\beta_{0}\right)=\frac{v^{\prime}\left(M_{1}-M\right) v / k_{2}}{v^{\prime} M v /(T-k)}
$$

and the conditional distribution of $A R\left(\beta_{0}\right)$ given $X$ only depends on $X$ and the distribution of $v$.

The proof is given in Appendix. This proposition means that the conditional null distribution of $A R\left(\beta_{0}\right)$, given $X$, only depends on the distribution of $v$. The distribution of $V$ plays no role here, so no decomposition assumption [such as $\mathrm{A}$ or $\mathrm{B}$ ] is needed. If the distribution of $v \mid X$ can be simulated, one can get exact tests based on $A R\left(\beta_{0}\right)$ through the Monte Carlo test method [see Dufour (2006)], even if this conditional distribution is non-Gaussian. Furthermore, the exact test obtained in this way is robust to weak instruments as well as instrument exclusion even if the distribution of $u \mid X$ does not have moments (e.g., the Cauchy distribution). ${ }^{3}$ This may be useful in financial models with fat-tailed error distributions, such as the Student $t$ distribution.

\footnotetext{
${ }^{3}$ By "robustness to weak instruments", we mean the fact that the null distribution of the test statistic remains valid even if $\operatorname{rank}\left[\Pi_{2}\right]<G$, so $\beta$ may not be identifiable from the available data. By "robustness to excluded instruments", we mean that the test remains valid even if $Y$ depends on additional explanatory variables $\left(X_{3}\right)$ which are not taken in IV-based inference; for further discussion of this issue, see Dufour and Taamouti (2007). Of course, identification failure (or weak identification) typically affects test power and confidence set precision. For example, if identification fails
} 
When the normality assumption holds $\left(v \sim \mathrm{N}\left[0, I_{T}\right]\right)$ and $X$ is exogenous, we have $A R\left(\beta_{0}\right) \sim F\left(k_{2}, T-k\right)$, so that $H_{\beta}\left(\beta_{0}\right)$ can be assessed by using a critical region of the form $\left\{A R\left(\beta_{0}\right)>f(\alpha)\right\}$, where $f(\alpha)=F_{\alpha}\left(k_{2}, T-k\right)$ is the $(1-\alpha)$-quantile of the $F\left(k_{2}, T-k\right)$ distribution. A confidence set with level $1-\alpha$ for $\beta$ is then given by

$$
\mathscr{C}_{\beta}(\alpha)=\left\{\beta_{0}: A R\left(\beta_{0}\right) \leq F_{\alpha}\left(k_{2}, T-k\right)\right\}=\{\beta: Q(\beta) \leq 0\}
$$

where $Q(\beta)=\beta^{\prime} A \beta+b^{\prime} \beta+c, A=Y^{\prime} H Y, b=-2 Y^{\prime} H y, c=y^{\prime} H y, H=M_{1}-\left[1+f(\alpha)\left(\frac{k_{2}}{T-k}\right)\right] M$, and $f(\alpha)=F_{\alpha}\left(k_{2}, T-k\right)$; see Dufour and Taamouti (2005).

\subsection{Inference on $\theta$}

Let us now consider the problem of testing the hypothesis $H_{\theta}\left(\theta_{0}\right): \theta=\theta_{0}$, where $\theta_{0}$ is a given vector of dimension $G$, and Assumption 3.2 holds. This can be done by considering the extended reduced form in (2.15). By Assumption 3.2, $e$ is independent of $Y, X_{1}$ and $X_{2}$, and (2.15) is a linear regression model. As soon as the matrix $\left[Y, X_{1}, X_{2}\right]$ has full-column rank, the parameters of equation (2.15) can be tested through standard $F$-tests.

We will now assume that $\left[Y, X_{1}, X_{2}\right]$ has full-column rank with probability one. This property holds as soon as $X=\left[X_{1}, X_{2}\right]$ has full column rank and $Y$ has a continuous distribution (conditional on $X)$. The $F$-statistic for testing $H_{\theta}\left(\theta_{0}\right)$ is

$$
F_{\theta}\left(\theta_{0}\right)=\frac{\left(\hat{\theta}-\theta_{0}\right)^{\prime}\left(Y^{\prime} M Y\right)\left(\hat{\theta}-\theta_{0}\right) / G}{y^{\prime} M(Z) y /(T-G-k)}
$$

where $\hat{\theta}=\left(Y^{\prime} M Y\right)^{-1} Y^{\prime} M y$ is the OLS estimate of $\theta$ in (2.15), $M=M(X), X=\left[X_{1}, X_{2}\right]$, and $Z=$ $\left[Y, X_{1}, X_{2}\right]$. When $v \sim \mathrm{N}\left[0, I_{T}\right]$, we have: $F_{\theta}\left(\theta_{0}\right) \sim F(G, T-k-G)$ under $H_{\theta}\left(\theta_{0}\right)$. Under the more general assumption 3.2 , it is easy to see that

$$
F_{\theta}\left(\theta_{0}\right)=\frac{\varepsilon^{\prime} M Y\left(Y^{\prime} M Y\right)^{-1} Y^{\prime} M \varepsilon / G}{\varepsilon^{\prime} M(Z) \varepsilon /(T-G-k)}
$$

under $H_{\theta}\left(\theta_{0}\right)$. On observing that the conditional distribution of $F_{\theta}\left(\theta_{0}\right)$, given $Y$ and $X$, does not involve any nuisance parameter, the critical value can be obtained by simulation. It is also important to note that this distribution does not depend on $\theta_{0}$, so the same critical value can be applied irrespective of $\theta_{0}$. The main difference with the Gaussian case is that the critical value may depend on $Y$ and $X$. Irrespective of the case, we shall denote by $c\left(\alpha_{2}\right)$ the critical value for $F_{\theta}\left(\theta_{0}\right)$.

From (3.6), a confidence set with level $1-\alpha$ for $\theta$ can be obtained by inverting $F_{\theta}\left(\theta_{0}\right)$ :

$$
\mathscr{C}_{\theta}(\alpha)=\left\{\theta_{0}: F_{\theta}\left(\theta_{0}\right) \leq \bar{f}(\alpha)\right\}=\left\{\theta_{0}: \bar{Q}\left(\theta_{0}\right) \leq 0\right\}
$$

where $\bar{Q}(\theta)=(\hat{\theta}-\theta)^{\prime}\left(Y^{\prime} M Y\right)(\hat{\theta}-\theta)-\bar{c}_{0}=\theta^{\prime} \bar{A} \theta+\bar{b}^{\prime} \theta+\bar{c}, \bar{c}_{0}=\bar{f}(\alpha) G s^{2}, s^{2}=y^{\prime} M(Z) y /(T-$

completely $\left(\operatorname{rank}\left[\Pi_{2}\right]=0\right)$, it is impossible to distinguish between alternative values of $\beta$, and a valid test of $H_{\beta}\left(\beta_{0}\right)$ should have power not larger than its level. Further, confidence sets of unidentified parameters should be uninformative (e.g., unbounded) with high probability; see Dufour (1997). 
$G-k), \bar{A}=Y^{\prime} M Y, \bar{b}=-2 \bar{A} \hat{\theta}=-2 Y^{\prime} M y, \bar{c}=\hat{\theta}^{\prime} \bar{A} \hat{\theta}-\bar{c}_{0}=\hat{\theta}^{\prime}\left(Y^{\prime} M Y\right) \hat{\theta}-\bar{c}_{0}=y^{\prime} \tilde{H} y$, and $\bar{H}=$ $P(M Y)-\bar{f}(\alpha)[G /(T-G-k)] M_{1}$. Since the matrix $\bar{A}$ is positive definite (with probability one), the quadric set $\mathscr{C}_{\theta}(\alpha)$ is an ellipsoid (hence bounded); see Dufour and Taamouti $(2005,2007)$. This reflects the fact that $\theta$ is an identifiable parameter. As a result, the corresponding projection-based confidence sets for scalar transformations $w^{\prime} \theta$ are also bounded intervals.

In view of the form (2.15) as a linear regression, we can test in the same way linear restrictions of the form $H_{w^{\prime} \theta}\left(\gamma_{0}\right): w^{\prime} \theta=\gamma_{0}$, where $w$ is a $G \times 1$ vector and $\gamma_{0}$ is known constant. We can then use the corresponding $t$ statistic

$$
t_{w^{\prime} \theta}\left(\gamma_{0}\right)=\frac{w^{\prime} \hat{\theta}-\gamma_{0}}{s\left[w^{\prime}\left(Y^{\prime} M Y\right)^{-1} w\right]^{1 / 2}}
$$

and reject $H_{w^{\prime} \theta}\left(\gamma_{0}\right)$ when $\left|t_{w^{\prime} \theta}\left(\gamma_{0}\right)\right|>c_{w}(\alpha)$, where $c_{w}(\alpha)$ is the critical value for a test with level $\alpha$. In the Gaussian case, $t_{w^{\prime} \theta}\left(\gamma_{0}\right)$ follows a Student distribution with $T-G-k$ degrees of freedom, so we can take $c_{w}(\alpha)=t\left(\alpha_{2} ; T-G-k\right)$. When $\varepsilon$ follows a non-Gaussian distribution, we have

$$
t_{w^{\prime} \theta}\left(\gamma_{0}\right)=\frac{(T-G-k)^{1 / 2} w^{\prime}\left(Y^{\prime} M Y\right)^{-1} Y^{\prime} M \varepsilon}{\left(\varepsilon^{\prime} M(Z) \varepsilon\right)^{1 / 2}\left[w^{\prime}\left(Y^{\prime} M Y\right)^{-1} w\right]^{1 / 2}}
$$

under $H_{w^{\prime} \theta}\left(\gamma_{0}\right)$, so that the distribution of $t_{w^{\prime} \theta}\left(\gamma_{0}\right)$ can be simulated like $F_{\theta}\left(\theta_{0}\right)$ in (3.7).

\subsection{Joint inference on $\beta$ and regression endogeneity parameters}

We can now derive confidence sets for the vectors $\left(\beta^{\prime}, a^{\prime}\right)^{\prime}$ and $\left(\beta^{\prime}, \theta^{\prime}\right)^{\prime}$. Consider the set:

$\mathscr{C}_{(\beta, \theta)}\left(\alpha_{1}, \alpha_{2}\right)=\left\{\left(\theta_{0}^{\prime}, \beta_{0}^{\prime}\right)^{\prime}: \beta_{0} \in \mathscr{C}_{\beta}\left(\alpha_{1}\right), \theta_{0} \in \mathscr{C}_{\theta}\left(\alpha_{2}\right)\right\}=\left\{\left(\theta_{0}^{\prime}, \beta_{0}^{\prime}\right)^{\prime}: Q\left(\beta_{0}\right) \leq 0, \bar{Q}\left(\theta_{0}\right) \leq 0\right\}$.

By the Boole-Bonferroni inequality, we have:

$$
\mathbb{P}\left[\beta \in \mathscr{C}_{\beta}\left(\alpha_{1}\right) \text { and } \theta \in \mathscr{C}_{\theta}\left(\alpha_{2}\right)\right] \geq 1-\mathbb{P}\left[\beta \notin \mathscr{C}_{\beta}\left(\alpha_{1}\right)\right]-\mathbb{P}\left[\theta \notin \mathscr{C}_{\theta}\left(\alpha_{2}\right)\right] \geq 1-\alpha_{1}-\alpha_{2}
$$

so $\mathscr{C}_{(\beta, \theta)}\left(\alpha_{1}, \alpha_{2}\right)$ is a confidence set for $\left(\beta^{\prime}, \theta^{\prime}\right)^{\prime}$ with level $1-\alpha$, where $\alpha=\alpha_{1}+\alpha_{2}$. In view of the identity $\theta=\beta+a$, we can write $\bar{Q}(\theta)$ in (3.8) as a function of $\beta$ and $a$ :

$$
\bar{Q}(\theta)=\bar{Q}(\beta+a)=a^{\prime} \bar{A} a+(\bar{b}+2 \bar{A} \beta)^{\prime} a+\left[\bar{c}+\bar{b}^{\prime} \beta+\beta^{\prime} \bar{A} \beta\right],
$$

so that we get a confidence set with level $1-\alpha$ for $\beta$ and $a$ by taking

$$
\overline{\mathscr{C}}_{(\beta, a)}(\alpha)=\left\{\left(\beta_{0}^{\prime}, a_{0}^{\prime}\right)^{\prime}: Q\left(\beta_{0}\right) \leq 0 \text { and } \bar{Q}\left(\beta_{0}+a_{0}\right) \leq 0\right\}
$$

Thus, finite-sample inference on the structural (possibly unidentifiable) parameter $a$ can be achieved. Of course, if $a$ is not identified, a valid confidence set will cover the set of all possible values (or be unbounded) with probability $1-\alpha$ [see Dufour (1997)]. 


\subsection{Confidence sets for regression endogeneity parameters}

We can now build "marginal" confidence sets for the endogeneity coefficient vector $a$. In view of the possibility of identification failure, this is most easily done by projection techniques. Let $g(\beta, a)$ be any function of $\beta$ and $a$. Since the event $(\beta, a) \in \overline{\mathscr{C}}_{(\beta, a)}(\alpha)$ entails $g(\beta, a) \in g\left[\overline{\mathscr{C}}_{(\beta, a)}(\alpha)\right]$, where $g\left[\overline{\mathscr{C}}_{(\beta, a)}(\alpha)\right]=\left\{g(\beta, a):(\beta, a) \in \overline{\mathscr{C}}_{(\beta, a)}(\alpha)\right\}$, we have:

$$
\mathbb{P}\left[g(\beta, a) \in g\left[\overline{\mathscr{C}}_{(\beta, a)}(\alpha)\right] \geq \mathbb{P}\left[(\beta, a) \in \overline{\mathscr{C}}_{(\beta, a)}(\alpha)\right] \geq 1-\alpha .\right.
$$

On taking $g(\beta, a)=a \in \mathbb{R}^{G}$, we see that

$$
\mathscr{C}_{a}(\alpha)=\left\{a:(\beta, a) \in \overline{\mathscr{C}}_{(\beta, a)}(\alpha) \text { for some } \beta\right\}=\{a: \bar{Q}(\beta+a) \leq 0 \text { and } Q(\beta) \leq 0 \text { for some } \beta\}
$$

is a confidence set with level $1-\alpha$ for $a$.

When $G=1$, the matrices $A, \bar{A}, b, \bar{b}, c$ and $\bar{c}$ in (3.8) reduce to scalars, and the different confidence sets take the following simple forms:

$$
\begin{aligned}
\mathscr{C}_{\beta}\left(\alpha_{1}\right) & =\left\{\beta: A \beta^{2}+b \beta+c \leq 0\right\}, \quad \mathscr{C}_{\theta}\left(\alpha_{2}\right)=\left\{\theta: \bar{A} \theta^{2}+\bar{b} \theta+\bar{c} \leq 0\right\}, \\
\mathscr{C}_{a}(\alpha) & =\left\{a: A \beta^{2}+b \beta+c \leq 0, \bar{A} a^{2}+(\bar{b}+2 \bar{A} \beta) a+\left[\bar{c}+\bar{b} \beta+\bar{A} \beta^{2}\right] \leq 0\right\} .
\end{aligned}
$$

Closed forms for the sets $\mathscr{C}_{\beta}\left(\alpha_{1}\right)$ and $\mathscr{C}_{\theta}\left(\alpha_{2}\right)$ are easily derived by finding the roots of the secondorder polynomial equations $A \beta^{2}+b \beta+c=0$ and $\bar{A} \theta^{2}+\bar{b} \theta+\bar{c}=0$ [as in Dufour and Jasiak (2001)], while the set $\mathscr{C}_{a}(\alpha)$ can be obtained by finding the roots of the equation

$$
\bar{A} a^{2}+\bar{b}(\beta) a+\bar{c}(\beta)=0 \text { where } \bar{b}(\beta)=\bar{b}+2 \bar{A} \beta \text { and } \bar{c}(\beta)=\bar{c}+\bar{b} \beta+\bar{A} \beta^{2} \text {, for each } \beta \in \mathscr{C}_{\beta}\left(\alpha_{1}\right) \text {. }
$$

We shall now focus on building confidence sets for scalar linear transformations $g(a)=w^{\prime} a=$ $w^{\prime} \theta-w^{\prime} \beta$, where $w$ is a $G \times 1$ vector. Conceptually, the simplest approach consists in applying the projection method to $\mathscr{C}_{a}(\alpha)$, which yields the confidence set:

$$
\begin{aligned}
\mathscr{C}_{w^{\prime} a}(\alpha) & =g_{w}\left[\mathscr{C}_{a}(\alpha)\right]=\left\{d: d=w^{\prime} a \text { for some } a \in \mathscr{C}_{a}(\alpha)\right\} \\
& =\left\{d: d=w^{\prime} a, \bar{Q}(\beta+a) \leq 0 \text { and } Q(\beta) \leq 0 \text { for some } \beta\right\} .
\end{aligned}
$$

But it will be more efficient to exploit the linear structure of model (2.15), which allows one to build a confidence interval for $w^{\prime} \theta$.

Following Dufour and Taamouti $(2005,2007)$, confidence sets for $g_{w}(\beta)=w^{\prime} \beta$ and $g_{w}(\theta)=$ $g_{w}=w^{\prime} \theta$ can be derived from $\mathscr{C}_{\beta}\left(\alpha_{1}\right)$ and $\mathscr{C}_{\theta}\left(\alpha_{2}\right)$ as follows:

$$
\mathscr{C}_{w^{\prime} \beta}\left(\alpha_{1}\right) \equiv g_{w}\left[\mathscr{C}_{\beta}\left(\alpha_{1}\right)\right]=\left\{x_{1}: x_{1}=w^{\prime} \beta, Q(\beta) \leq 0\right\}=\left\{x_{1}: x_{1}=w^{\prime} \beta, \beta^{\prime} A \beta+b^{\prime} \beta+c \leq 0\right\}
$$

where $A, b$ and $c$ are defined as in (3.5). For $w^{\prime} \theta$, we can use a $t$-type confidence interval based on $t\left(\gamma_{0}\right):$

$$
\overline{\mathscr{C}}_{w^{\prime} \theta}\left(\alpha_{2}\right) \equiv \bar{g}_{w}\left[\mathscr{C}_{\theta}\left(\alpha_{2}\right)\right]=\left\{\gamma_{0}:\left|t_{w^{\prime} \theta}\left(\gamma_{0}\right)\right|<c_{w}\left(\alpha_{2}\right)\right\}=\left\{\gamma_{0}:\left|w^{\prime} \hat{\theta}-\gamma_{0}\right|<\bar{D}\left(\alpha_{2}\right)\right\}
$$


where $\bar{D}\left(\alpha_{2}\right)=c_{w}\left(\alpha_{2}\right) \hat{\sigma}\left(w^{\prime} \hat{\theta}\right), \hat{\sigma}\left(w^{\prime} \hat{\theta}\right)=s\left[w^{\prime}\left(Y^{\prime} M Y\right)^{-1} w\right]^{1 / 2}$ with $s=\left[y^{\prime} M(Z) y\right]^{1 / 2} /(T-G-$ $k)^{1 / 2}$, and $c_{w}\left(\alpha_{2}\right)$ is the critical value for a test with level $\alpha_{2}$ based on $t_{w^{\prime} \theta}\left(\gamma_{0}\right)$ [in (3.9)]. Setting

$$
\mathscr{C}_{\left(w^{\prime} \beta, w^{\prime} \theta\right)}\left(\alpha_{1}, \alpha_{2}\right)=\left\{(x, y)^{\prime}: x \in \mathscr{C}_{w^{\prime} \beta}\left(\alpha_{1}\right) \text { and } y \in \overline{\mathscr{C}}_{w^{\prime} \theta}\left(\alpha_{2}\right)\right\},
$$

we see that $\mathscr{C}_{\left(w^{\prime} \beta, w^{\prime} \theta\right)}\left(\alpha_{1}, \alpha_{2}\right)$ is a confidence set for $\left(w^{\prime} \beta, w^{\prime} \theta\right)$ with level $1-\alpha_{1}-\alpha_{2}$ :

$$
\mathbb{P}\left[\left(w^{\prime} \beta, w^{\prime} \theta\right) \in \mathscr{C}_{\left(w^{\prime} \beta, w^{\prime} \theta\right)}\left(\alpha_{1}, \alpha_{2}\right)\right]=\mathbb{P}\left[w^{\prime} \beta \in \mathscr{C}_{w^{\prime} \beta}\left(\alpha_{1}\right) \text { and } w^{\prime} \theta \in \overline{\mathscr{C}}_{w^{\prime} \theta}\left(\alpha_{2}\right)\right] \geq 1-\alpha
$$

where $\alpha=\alpha_{1}+\alpha_{2}$. For any point $x \in \mathbb{R}$ and any subset $A \subseteq \mathbb{R}$, set $x-A=\{z \in \mathbb{R}: z=x-y$ and $y \in$ $A\}$. Since $w^{\prime} a=w^{\prime} \theta-w^{\prime} \beta$, it is clear that

$$
\begin{gathered}
\left(w^{\prime} \beta, w^{\prime} \theta\right) \in \mathscr{C}_{\left(w^{\prime} \beta, w^{\prime} \theta\right)}\left(\alpha_{1}, \alpha_{2}\right) \Leftrightarrow w^{\prime} \theta-w^{\prime} a \in \mathscr{C}_{w^{\prime} \beta}\left(\alpha_{1}\right) \text { and } w^{\prime} \theta \in \overline{\mathscr{C}}_{w^{\prime} \theta}\left(\alpha_{2}\right) \\
\Leftrightarrow w^{\prime} a \in w^{\prime} \theta-\mathscr{C}_{w^{\prime} \beta}\left(\alpha_{1}\right) \text { and } w^{\prime} \theta \in \overline{\mathscr{C}}_{w^{\prime} \theta}\left(\alpha_{2}\right), \\
\mathbb{P}\left[w^{\prime} a \in w^{\prime} \theta-\mathscr{C}_{w^{\prime} \beta}\left(\alpha_{1}\right) \text { and } w^{\prime} \theta \in \overline{\mathscr{C}}_{w^{\prime} \theta}\left(\alpha_{2}\right)\right] \\
\quad=\mathbb{P}\left[w^{\prime} \beta \in \mathscr{C}_{w^{\prime} \beta}\left(\alpha_{1}\right) \text { and } w^{\prime} \theta \in \overline{\mathscr{C}}_{w^{\prime} \theta}\left(\alpha_{2}\right)\right] \\
\\
\geq 1-\alpha_{1}-\alpha_{2} .
\end{gathered}
$$

Now, consider the set

$$
\mathscr{C}_{w^{\prime} a}\left(\alpha_{1}, \alpha_{2}\right)=\left\{z \in \mathbb{R}: z \in y-\mathscr{C}_{w^{\prime} \beta}\left(\alpha_{1}\right) \text { for some } y \in \overline{\mathscr{C}}_{w^{\prime} \theta}\left(\alpha_{2}\right)\right\} .
$$

Since the event $\left\{w^{\prime} a \in w^{\prime} \theta-\mathscr{C}_{w^{\prime} \beta}\left(\alpha_{1}\right)\right.$ and $\left.w^{\prime} \theta \in \overline{\mathscr{C}}_{w^{\prime} \theta}\left(\alpha_{2}\right)\right\}$ entails $w^{\prime} a \in \mathscr{C}_{w^{\prime} a}\left(\alpha_{1}, \alpha_{2}\right)$, we have:

$$
\mathbb{P}\left[w^{\prime} a \in \mathscr{C}_{w^{\prime} a}\left(\alpha_{1}, \alpha_{2}\right)\right] \geq \mathbb{P}\left[w^{\prime} \beta \in \mathscr{C}_{w^{\prime} \beta}\left(\alpha_{1}\right) \text { and } w^{\prime} \theta \in \overline{\mathscr{C}}_{w^{\prime} \theta}\left(\alpha_{2}\right)\right] \geq 1-\alpha_{1}-\alpha_{2}
$$

and $\mathscr{C}_{w^{\prime} a}\left(\alpha_{1}, \alpha_{2}\right)$ is a confidence set with level $1-\alpha_{1}-\alpha_{2}$ for $w^{\prime} a$.

Since $\overline{\mathscr{C}}_{w^{\prime} \theta}\left(\alpha_{2}\right)$ is a bounded interval, the shape of $\mathscr{C}_{w^{\prime} a}\left(\alpha_{1}, \alpha_{2}\right)$ can be deduced easily by using the results given in Dufour and Taamouti $(2005,2007)$. We focus on the case where $A$ is nonsingular [an event with probability one as soon as the distribution of $A R\left(\beta_{0}\right)$ is continuous] and $w \neq 0$. Then the set $\mathscr{C}_{w^{\prime} \beta}\left(\alpha_{1}\right)$ may then rewritten as follows: if $A$ is positive definite,

$$
\begin{aligned}
\mathscr{C}_{w^{\prime} \beta}\left(\alpha_{1}\right) & =\left[w^{\prime} \tilde{\beta}-D\left(\alpha_{1}\right), w^{\prime} \tilde{\beta}+D\left(\alpha_{1}\right)\right], & & \text { if } d \geq 0, \\
& =\emptyset, & & \text { if } d<0
\end{aligned}
$$

where $\tilde{\beta}=-\frac{1}{2} A^{-1} b, d=\frac{1}{4} b^{\prime} A^{-1} b-c$ and $D\left(\alpha_{1}\right)=\sqrt{d\left(w^{\prime} A^{-1} w\right)}$; if $A$ has exactly one negative eigenvalue and $d<0$,

$$
\begin{aligned}
\mathscr{C}_{w^{\prime} \beta}\left(\alpha_{1}\right) & \left.=]-\infty, w^{\prime} \tilde{\beta}-D\left(\alpha_{1}\right)\right] \cup\left[w^{\prime} \tilde{\beta}+D\left(\alpha_{1}\right),+\infty[,\right. & & \text { if } w^{\prime} A^{-1} w<0, \\
& =\mathbb{R} \backslash\left\{w^{\prime} \tilde{\beta}\right\}, & & \text { if } w^{\prime} A^{-1} w=0 ;
\end{aligned}
$$

otherwise, $\mathscr{C}_{w^{\prime} \beta}\left(\alpha_{1}\right)=\mathbb{R}$. $\mathscr{C}_{w^{\prime} \beta}\left(\alpha_{1}\right)=\emptyset$ corresponds to a case where the model is not consistent with the data [so that $\mathscr{C}_{w^{\prime} a}\left(\alpha_{1}, \alpha_{2}\right)=\emptyset$ as well], while $\mathscr{C}_{w^{\prime} \beta}\left(\alpha_{1}\right)=\mathbb{R}$ and $\mathscr{C}_{w^{\prime} \beta}\left(\alpha_{1}\right)=\mathbb{R} \backslash\left\{w^{\prime} \tilde{\beta}\right\}$ indicate that $w^{\prime} \beta$ is not identifiable and similarly for $w^{\prime} a$ [so that $\mathscr{C}_{w^{\prime} a}\left(\alpha_{1}, \alpha_{2}\right)=\mathbb{R}$ ]. This yields the 
following confidence sets for $w^{\prime} a$ : if $A$ is positive definite,

$$
\begin{aligned}
\mathscr{C}_{w^{\prime} a}\left(\alpha_{1}, \alpha_{2}\right) & =\left[w^{\prime}(\hat{\theta}-\tilde{\beta})-D_{U}\left(\alpha_{1}, \alpha_{2}\right), w^{\prime}(\hat{\theta}-\tilde{\beta})+D_{U}\left(\alpha_{1}, \alpha_{2}\right)\right], & & \text { if } d \geq 0, \\
& =\emptyset, & & \text { if } d<0,
\end{aligned}
$$

where $D_{U}\left(\alpha_{1}, \alpha_{2}\right)=D\left(\alpha_{1}\right)+\bar{D}\left(\alpha_{2}\right)$; if $A$ has exactly one negative eigenvalue, $w^{\prime} A^{-1} w<0$ and $d<0$,

$$
\left.\left.\mathscr{C}_{w^{\prime} a}\left(\alpha_{1}, \alpha_{2}\right)=\right]-\infty, w^{\prime}(\hat{\theta}-\tilde{\beta})-D_{L}\left(\alpha_{1}, \alpha_{2}\right)\right] \cup\left[w^{\prime}(\hat{\theta}-\tilde{\beta})+D_{L}\left(\alpha_{1}, \alpha_{2}\right),+\infty[\right.
$$

where $D_{L}\left(\alpha_{1}, \alpha_{2}\right)=D\left(\alpha_{1}\right)-\bar{D}\left(\alpha_{2}\right)$; otherwise, $\mathscr{C}_{w^{\prime} a}\left(\alpha_{1}, \alpha_{2}\right)=\mathbb{R}$. These results may be extended to cases where $A$ is singular, as done by Dufour and Taamouti (2007).

\subsection{Exact Monte Carlo identification-robust tests with non-Gaussian errors}

Suppose now that the conditional distribution of $v$ (given $X$ ) is continuous, so that the conditional distribution of $A R\left(\beta_{0}\right)$ under the null hypothesis $H_{\beta}\left(\beta_{0}\right)$ is also continuous. We can then proceed as follows to obtain an exact Monte Carlo test of $H_{\beta}\left(\beta_{0}\right)$ with level $\alpha(0<\alpha<1)$ :

(1) choose $\alpha^{*}$ and $N$ so that $\alpha=\left(I\left[\alpha^{*} N\right]+1\right) /(N+1)$;

(2) for given $\beta_{0}$, compute the test statistic $A R^{(0)}\left(\beta_{0}\right)$ based on the observed data;

(3) generate $N$ i.i.d. error vectors $v^{(j)}=\left[v_{1}^{(j)}, \ldots, v_{T}^{(j)}\right]^{\prime}, j=1, \ldots, N$, according to the specified distribution of $v \mid X$, and compute the corresponding statistic $A R^{(j)}, j=1, \ldots, N$, following (3.4); note the distribution of $A R\left(\beta_{0}\right)$ does not depend on the specific value $\beta_{0}$ tested, so there is no need to make it depend on $\beta_{0}$;

(4) compute the simulated $p$-value function: $\hat{p}_{N}[x]=\left\{1+\sum_{j=1}^{N} \mathbb{1}\left[A R^{(j)} \geq x\right]\right\} /(N+1)$, where $\mathbb{1}[C]=1$ if condition $C$ holds, and $\mathbb{1}[C]=0$ otherwise;

(5) reject the null hypothesis $H_{\beta}\left(\beta_{0}\right)$ at level $\alpha$ when $\hat{p}_{N}\left[A R^{(0)}\left(\beta_{0}\right)\right] \leq \alpha$.

Under the null hypothesis $H_{\beta}\left(\beta_{0}\right), \mathbb{P}\left[\hat{p}_{N}\left[A R^{(0)}\left(\beta_{0}\right)\right] \leq \alpha\right]=\alpha$, so that we have a test with level $\alpha$. If the distribution of the test statistic is not continuous, the MC test procedure can easily be adapted by using "tie-breaking" method described in Dufour (2006). ${ }^{4}$ Correspondingly, a confidence set with level $1-\alpha$ for $\beta$ is given by the set of all values $\beta_{0}$ which are not rejected by the above MC test. More precisely, the set

$$
\mathscr{C}_{\beta}(\alpha)=\left\{\beta_{0}: \hat{p}_{N}\left[A R^{(0)}\left(\beta_{0}\right)\right]>\alpha\right\}
$$

is a confidence set with level $1-\alpha$ for $\beta$. On noting that the distribution of $A R\left(\beta_{0}\right)$ does not depend on $\beta_{0}$, we can use a single simulation for all values $\beta_{0}$ : setting $\hat{f}_{N}\left(\alpha^{*}\right)=\hat{F}_{N}^{-1}\left(1-\alpha^{*}\right)$, the set

$$
\mathscr{C}_{\beta}(\alpha ; N)=\left\{\beta_{0}: A R^{(0)}<\hat{f}_{N}\left(\alpha^{*}\right)\right\}
$$

\footnotetext{
${ }^{4}$ Without the correction for continuity, the algorithm proposed for statistics with continuous distributions yields a conservative test, i.e. the probability of rejection under the null hypothesis is not larger than the nominal level $\left(\alpha_{1}\right)$.
} 
is equivalent to $\mathscr{C}_{\beta}(\alpha)$ - with probability one - and so has level $1-\alpha$. On replacing $>$ and $<$ by $\geq$ and $\leq$ in (3.24) - (3.25), it is also clear that the sets $\left\{\beta_{0}: \hat{p}_{N}\left[A R^{(0)}\left(\beta_{0}\right)\right] \geq \alpha\right\}$ and

$$
\overline{\mathscr{C}}_{\beta}(\alpha ; N)=\left\{\beta_{0}: A R^{(0)}\left(\beta_{0}\right) \leq \hat{f}_{N}\left(\alpha^{*}\right)\right\}
$$

constitute confidence sets for $\beta$ with level $1-\alpha$ (though possibly a little larger than $1-\alpha$ ). The quadric form given in (3.5) also remains valid with $f(\alpha)=\hat{f}_{N}\left(\alpha^{*}\right)$.

\section{Asymptotic theory for inference on endogeneity parameters}

In this section, we examine the validity of the procedures developed in Section 3 under weaker distributional assumptions, and we show how inference on covariance endogeneity parameters can be made. On noting that equations (3.2) and (2.15) constitute standard linear regression models (at least under the null hypothesis $\beta=\beta_{0}$ ), it is straightforward to find high-level regularity conditions under which the tests based on $A R\left(\beta_{0}\right)$ and $F_{\theta}\left(\theta_{0}\right)$ are asymptotically valid.

For $A R\left(\beta_{0}\right)$, we can consider the following general assumption.

Assumption 4.1 When the sample size $T$ converges to infinity, the following convergence results hold jointly: (a) $\frac{1}{T} X^{\prime} u \stackrel{p}{\rightarrow} 0 ;(b) \frac{1}{T} u^{\prime} u \stackrel{p}{\rightarrow} \sigma_{u}^{2}>0, \frac{1}{T} X^{\prime} X \stackrel{p}{\rightarrow} \Sigma_{X}$ with $\operatorname{det}\left(X^{\prime} X\right) \neq 0 ;(c) \frac{1}{\sqrt{T}} X^{\prime} u \stackrel{L}{\rightarrow}$ $\psi_{X u}, \psi_{X u} \sim N\left[0, \sigma_{u}^{2} \Sigma_{X}\right]$, where $X=\left[X_{1}, X_{2}\right]$.

The above conditions are easy to interpret: (a) represents the asymptotic orthogonality between $u$ and the instruments in $X$, (b) may be viewed as laws of large numbers for $u$ and $X$, while (c) is a central limit property. Then, it is a simple exercise to see that

$$
A R\left(\beta_{0}\right) \stackrel{L}{\rightarrow} \chi^{2}\left(k_{2}\right) / k_{2}, \quad \text { when } \beta=\beta_{0} .
$$

Similarly, for $F_{\theta}\left(\theta_{0}\right)$, we can suppose the following.

Assumption 4.2 When the sample size $T$ converges to infinity, the following convergence results hold jointly: (a) $\frac{1}{T} Z^{\prime} e \stackrel{p}{\rightarrow} 0 ;(b) \frac{1}{T} e^{\prime} e \stackrel{p}{\rightarrow} \sigma_{e}^{2}>0, \frac{1}{T} Z^{\prime} Z \stackrel{p}{\rightarrow} \Sigma_{Z}$ with $\operatorname{det}\left(Z^{\prime} Z\right) \neq 0 ;(c) \frac{1}{\sqrt{T}} Z^{\prime} e \stackrel{L}{\rightarrow}$ $\psi_{Z e}, \psi_{Z e} \sim N\left[0, \sigma_{e}^{2} \Sigma_{Z}\right]$, where $Z=\left[Y, X_{1}, X_{2}\right]$.

Then

$$
F_{\theta}\left(\theta_{0}\right) \stackrel{L}{\rightarrow} \chi^{2}(G) / G, \quad \text { when } \theta=\theta_{0} .
$$

The asymptotic distributions in (4.1) and (4.2) hold irrespective whether the instruments $X$ are weak or strong. Further, as soon as assumptions 4.1 and 4.2 hold, the confidence procedures described in Section 3 remain "asymptotically valid" with $f\left(\alpha_{1}\right)=\chi^{2}\left(\alpha_{1} ; k_{2}\right) / k_{2}$ and $\bar{f}\left(\alpha_{2}\right)=\chi^{2}\left(\alpha_{2} ; G\right) / G$, where $\chi^{2}\left(\alpha_{1} ; k_{2}\right)$ and $\chi^{2}\left(\alpha_{2} ; G\right)$ are respectively the $1-\alpha_{1}$ and $1-\alpha_{2}$ quantiles of the corresponding $\chi^{2}$ distributions. Of course, the Gaussian-based Fisher critical values may also be used (for they converge to the chi-square critical values as $T \rightarrow \infty$ ).

We can now consider inference for covariance endogeneity parameters $\sigma_{V u}$. The problem of building confidence sets for $\sigma_{V u}$ is especially important for assessing partial exogeneity hypotheses. 
Since $a_{j}=0, j=1, \ldots, G$ does not entail $\sigma_{u V j}=0$ (where $\left.1 \leq j \leq G\right)$, confidence sets on the components of $a$ cannot directly be used to assess for example, the exogeneity of each regressor $Y_{j}, j=1, \ldots, G$. Confidence sets and tests for $\sigma_{u V}$ can be deduced from those on $a$ through the relationship $\sigma_{V u}=\Sigma_{V} a$ given in (2.9). On replacing $a$ by $\Sigma_{V}^{-1} \sigma_{V u}$ in $\mathscr{C}_{a}(\alpha)$, we see that the set

$$
\begin{aligned}
\mathscr{C}_{\sigma_{V u}}\left(\alpha ; \Sigma_{V}\right) & =\left\{\sigma_{V u} \in \mathbb{R}^{G}: \sigma_{V u}=\Sigma_{V} a \text { and } a \in \mathscr{C}_{a}(\alpha)\right\} \\
& =\left\{\sigma_{V u} \in \mathbb{R}^{G}: \bar{Q}\left(\beta+\Sigma_{V}^{-1} \sigma_{V u}\right) \leq 0 \text { and } Q(\beta) \leq 0 \text { for some } \beta\right\}
\end{aligned}
$$

is a confidence set with level $1-\alpha$ for $\sigma_{V u}$. This set is simply the image of $\mathscr{C}_{a}(\alpha)$ by the linear transformation $g(x)=\Sigma_{V} x$. The difficulty here comes from the fact that $\Sigma_{V}$ is unknown. Let $\hat{\Sigma}_{V}=$ $\hat{V}^{\prime} \hat{V} /(T-k)$ where $\hat{V}=M(X) Y$ is the matrix of least-squares residuals from the first-step regression (2.2). Under standard regularity conditions, we have:

$$
\hat{\Sigma}_{V} \stackrel{p}{\rightarrow} \Sigma_{V}
$$

where $\operatorname{det}\left(\Sigma_{V}\right)>0$. If $\beta_{0}$ and $a_{0}$ are the true values of $\beta$ and $a$, the relations $\theta_{0}=\beta_{0}+a_{0}$ and $\sigma_{V u 0}=\Sigma_{V} a_{0}$ entail that $F_{\theta}\left(\theta_{0}\right)$ can be rewritten as follows:

$$
F_{\theta}\left(\beta_{0}+\Sigma_{V}^{-1} \sigma_{V u 0}\right)=\frac{\left(\hat{\theta}-\beta_{0}-\Sigma_{V}^{-1} \sigma_{V u 0}\right)^{\prime}\left(Y^{\prime} M Y\right)\left(\hat{\theta}-\beta_{0}-\Sigma_{V}^{-1} \sigma_{V u 0}\right) / G}{y^{\prime} M(Z) y /(T-G-k)} .
$$

Replacing $\Sigma_{V}$ by $\hat{\Sigma}_{V}$, we get the approximate pivotal function $F_{\theta}\left(\beta_{0}+\hat{\Sigma}_{V}^{-1} \sigma_{V u 0}\right)$. If (4.4) holds, it is easy to see (by continuity) that $F_{\theta}\left(\beta_{0}+\hat{\Sigma}_{V}^{-1} \sigma_{V u 0}\right)$ and $F_{\theta}\left(\beta_{0}+\Sigma_{V}^{-1} \sigma_{V u 0}\right)$ are asymptotically equivalent with a nondegenerate distribution, when $\beta_{0}$ and $\sigma_{V u 0}$ are the true parameter values. Consequently, the confidence set of type $\mathscr{C}_{\sigma_{V u}}(\alpha)$ based on $F_{\theta}\left(\beta_{0}+\hat{\Sigma}_{V}^{-1} \sigma_{V u 0}\right)$ as opposed to $F_{\theta}\left(\beta_{0}+\Sigma_{V}^{-1} \sigma_{V u 0}\right)$ has level $1-\alpha$ asymptotically. This set is simply the image of $\mathscr{C}_{a}(\alpha)$ by the linear transformation $\hat{g}(x)=\hat{\Sigma}_{V} x$, i.e.

$$
\mathscr{C}_{\sigma_{V u}}\left(\alpha ; \hat{\Sigma}_{V}\right)=\left\{\sigma_{V u} \in \mathbb{R}^{G}: \bar{Q}\left(\beta+\hat{\Sigma}_{V}^{-1} \sigma_{V u}\right) \leq 0 \text { and } Q(\beta) \leq 0 \text { for some } \beta\right\} .
$$

Finally, confidence sets for the components of $\sigma_{V u}$, and more generally for linear combinations $w^{\prime} \sigma_{V u}$, can be derived from those on $w^{\prime} a$ as described in Section 3.4. For $\Sigma_{V}$ given, the relation $\sigma_{V u}=\Sigma_{V} a$ entails that a confidence set for $w^{\prime} \sigma_{V u}$ (with level $1-\alpha$ ) can be obtained by computing a confidence set (at level $1-\alpha$ ) for $w_{1}^{\prime} a$ with $w_{1}=\Sigma_{V} w$. When $\Sigma_{V}$ is estimated by $\hat{\Sigma}_{V}$, taking $w_{1}=$ $\hat{\Sigma}_{V} w$ yields a confidence set for $\sigma_{V u}$ with level $1-\alpha$ asymptotically.

\section{Empirical applications}

We will now apply the methods proposed above to three empirical examples: a model of the relation between trade and economic growth, previously studied in Frankel and Romer (1999) and Dufour and Taamouti (2007); and two models of the returns to educations, the well-known study of Bound et al. (1995), and an alternative model considered by Card (1995) and Kleibergen (2004, Table 2, p. 421). 


\subsection{Trade and growth}

The trade and growth model studies the relationship between standards of living and openness. Frankel and Romer (1999) argued that trade share (ratio of imports or exports to GDP) which is the commonly used indicator of openness may be endogenous. The equation studied is given by:

$$
\ln \left(\text { Income }_{i}\right)=\beta_{0}+\beta \operatorname{Trade}_{i}+\gamma_{1} \ln \left(\text { Pop }_{i}\right)+\gamma_{2} \ln \left(\text { Area }_{i}\right)+u_{i}, i=1, \ldots, N
$$

where Income is the income per capita, Trade is measured as a ratio of imports and exports to GDP, Pop is the logarithm of the country population, and Area is the logarithm of the country area. The instrument suggested is constructed on the basis of geographic characteristics. The first stage equation is then given by:

$$
\operatorname{Trade}_{i}=b_{0}+b_{1} Z_{i}+c_{1} \operatorname{Pop}_{i}+c_{2} \mathrm{Area}_{i}+V_{i}, i=1, \ldots, N,
$$

where $Z_{i}$ is a constructed instrument. We use the sample of 150 countries and the data are for 1985 . Dufour and Taamouti (2005) showed that the fitted instrument in this sample is not very weak. ${ }^{5}$

The identification-robust confidence intervals with level $97.5 \%$ for $\beta$ and $\theta=\beta_{1}+a$, that result on inverting $A R\left(\beta_{0}\right)$ and $t_{\theta}\left(\gamma_{0}\right)$ are given by: $\mathscr{C}_{\beta}(\alpha)=\left\{\beta_{0}: 0.23 \beta_{0}^{2}-4.76 \beta_{0}+0.04 \leq 0\right\}=$ $[0.01,20.62]$ and $\mathscr{C}_{\theta}(\alpha)=[-0.05,0.47]$. The results reported are based on the critical values of the $F$-distributions of Section 3. The Monte Carlo method as described in Section 3.5 gives similar results even with 1000 replications. We see that $\mathscr{C}_{\beta}(\alpha)$ is a bounded interval, thus confirming that identification is not weak in this model. The estimates of regression and covariance endogeneity parameters are given by $\hat{a}=-1.82$ and $\hat{\sigma}_{u V}=-0.38$, respectively. The confidence intervals ${ }^{6}$ with level $95 \%$ for $a$ and $\sigma_{V u}$ are given by:

$$
\mathscr{C}_{a}(\alpha)=[-20.67,0.46] \text { and } \quad \mathscr{C}_{\sigma_{V u}}(\alpha)=[-4.33,0.09] .
$$

Both confidence intervals are bounded and contain the estimates of $a$ and $\sigma_{V u}$ from observed data. Both confidence intervals, though include zero, are left skewed at zero. In particular, the upper bound for $\mathscr{C}_{\sigma_{V u}}(\alpha)$ is very close to zero. So the true covariance and regression endogeneity parameters can be actually large, thus indicating the importance of omitting variables bias (for $a$ ) and trade share endogeneity (for $\sigma_{V u}$ ). The latter is likely plausible as the discrepancy between the OLS estimate of $\beta\left(\hat{\beta}_{\text {OLS }}=0.28\right)$ and the 2 SLS estimate $\left(\hat{\beta}_{2 S L S}=2.03\right)$ is relatively large.

\subsection{Angrist-Krueger model of education and earnings}

We now consider the problem of estimating the returns to schooling. The model studies a relationship between log weekly earning and the number of years of education and several other covariates (age, squared age, year of birth, ... ). Several authors including Angrist and Krueger (1991) argued that schooling may be endogenous in this model and proposed to use the birth quarter as an

\footnotetext{
${ }^{5}$ The $F$-statistic in the first stage (5.2) is about 13, see also Frankel and Romer (1999, Table 2, p.385).

${ }^{6}$ Note that the confidence interval with level $95 \%$ for $a$ and $\sigma_{V u}$, obtained on inverting $A R\left(\beta_{0}\right)$ and $F_{\theta}\left(\theta_{0}\right)$ are similar to those reported here.
} 
Table 1. Projection-based confidence sets for different parameters in earning equation

\begin{tabular}{|c|c|c|}
\hline$A R$-type CS's & $97.5 \%$ & $95 \%$ \\
$C_{\beta_{1}}(\alpha)$ & $\left\{\beta_{1}:-2.382 \beta_{1}^{2}+0.332 \beta_{1}-0.107 \leq 0\right\}$ & $\left\{\beta_{1}:-2.229 \beta_{1}^{2}+0.31 \beta_{1}-0.1 \leq 0\right\}$ \\
$C_{\theta}(\alpha)$ & $=\mathbb{R}$ & $=\mathbb{R}$ \\
& $\left\{\theta: 3.527 \theta^{2}-0.5 \theta+0.018 \leq 0\right\}$ & $\left\{\theta: 3.527 \theta^{2}-.5 \theta+0.018 \leq 0\right\}$ \\
$C_{\theta}(\alpha)$ based on $t_{w^{\prime} \theta}\left(\gamma_{0}\right)$ & $=[0.0701,0.0716]$ & $=[.0702, .0715]$ \\
Projection-based CS's & {$[.0707, .0710]$} & {$[.0707, .0710]$} \\
$\mathscr{C}_{a}(\alpha)$ & $95 \%$ & $90 \%$ \\
$C_{\sigma_{V u}}(\alpha)$ & $\mathbb{R}$ & $\mathbb{R}$ \\
& $\mathbb{R}$ & $\mathbb{R}$ \\
\hline
\end{tabular}

instrument to estimate the returns to schooling consistently. The reason is individuals born in the first quarter of the year start school at an older age, and can therefore drop out after completing less schooling than individuals born near the end of the year. Hence, individuals born at the beginning of the year are likely to earn less than those born during the rest of the year. Bound et al. (1995) however, showed that the quarter of birth instruments are very weak. In Doko Tchatoka and Dufour (2011b, 2011a), we show that DWH tests cannot detect the endogeneity of schooling in this model, since the instruments have poor quality [see Dufour and Taamouti (2007)].

Here, we assess whether schooling is exogenous by using the projection method developed in this paper. The model is specified by

$$
\begin{aligned}
y & =\beta_{0}+\beta_{1} E+\sum_{i=1}^{k_{1}} \gamma_{i} X_{i}+u, \\
E & =\pi_{0}+\sum_{i=1}^{k_{2}} \pi_{i} Z_{i}+\sum_{i=1}^{k_{1}} \phi_{i} X_{i}+V
\end{aligned}
$$

where $y$ is log-weekly earnings, $E$ is the number of years of education (possibly endogenous), $X$ contains the exogenous covariates (age, age squared, 10 dummies for birth of year). $Z$ contains 40 dummies obtained by interacting the quarter of birth with the year of birth. In this model, $\beta_{1}$ measures the return to education. The data set consists of the 5\% public-use sample of the 1980 US census for men born between 1930 and 1939. The sample size is 329509 observations.

Table 1 presents the results. We observe that $\mathscr{C}_{\beta}(\alpha)$ is unbounded indicating that $\beta$ is not identified. However, $\mathscr{C}_{\theta}(\alpha)$ is bounded The latter result confirms the fact that $\theta$ is always identified even if identification is weak (weak instrument). As a result, $\mathscr{C}_{a}(\alpha)$ and $\mathscr{C}_{\sigma_{V u}}(\alpha)$ are unbounded in all cases. That indicates clearly that identification is an issue in this model.

\subsection{Card model of education and earnings}

We will also apply the methods proposed to the following alternative model studied by Card (1995) for the return of education to earnings: 


$$
\begin{gathered}
y_{i}=Y_{1 i} \beta_{1}+Y_{2 i} \beta_{2}+Y_{3 i} \beta_{3}+X_{1 i}^{\prime} \gamma+u_{i}, \\
\left(Y_{1 i}, Y_{2 i}, Y_{3 i}\right)=X_{1 i}^{\prime} \Pi_{1}+X_{2 i}^{\prime} \Pi_{2}+V_{i},
\end{gathered}
$$

where $Y_{1 i}$ is the length of education of individual $i,\left(Y_{2 i}, Y_{3 i}\right)=\left(\right.$ exper $_{i}$, exper $\left._{i}^{2}\right)$ contains the experience (exper) and experience squared of individual $i$ where exper ${ }_{i}=\operatorname{age}_{i}-6-Y_{1 i} ; X_{1 i}=$ $\left(1, \text { race }_{i}, \text { smsa }_{i}, \text { south }_{i}\right)^{\prime}$ consists of a constant and indicator variables for race, residence in a metropolitan area, and residence in the south of the United States; and $y_{i}$ is the logarithm of the wage of individual $i$. All variables in $X_{1}$ are assumed exogenous. $X_{2 i}$ is the vector of instruments that contains age, age $e^{2}$ of individual $i$, and proximity-to-college indicators for educational attainment; these are proximity to 2- and 4-year college. Kleibergen (2004, Table 2, p. 421) shows that the proximity-to-college indicator instruments are not very strong. Hence, it is important to be careful when interpreting the 2SLS estimates of this model. We follow the methodology developed in this paper for building projection-based confidence intervals of the components of the regression and covariance endogeneity parameters $a=\left(a_{1}, a_{2}, a_{3}\right)^{\prime}$ and $\sigma_{V u}=\left(\sigma_{V u 1}, \sigma_{V u 2}, \sigma_{V u 3}\right)^{\prime}$.

The data analyzed are from the National Longitudinal Survey of Young Men (from 1966 to 1981). We use the cross-sectional 1976 subsample which contains 3010 observations. After accounting for missing data, the final sample has 2061 observations. The variables contained in the data set are: two variables indicating the proximity to college, the length of education, log wages, experience, IQ score, age, racial, metropolitan, family, and regional indicators.

To build confidence sets with level $95 \%$ for $a$ and $\sigma_{V u}$, we take $\alpha_{1}=\alpha_{2}=0.025$. The identification-robust confidence sets with level $97.5 \%$ for $\beta=\left(\beta_{1}, \beta_{2}, \beta_{3}\right)^{\prime}$ and $\theta=\beta+a$, based on inverting $A R\left(\beta_{0}\right)$ and $F_{\theta}\left(\theta_{0}\right)$ are given by: $\mathscr{C}_{\beta}(\alpha)=\left\{\beta_{0}: \beta_{0}^{\prime} A \beta_{0}-b^{\prime} \beta_{0}+0.37 \leq 0\right\}$ and $\mathscr{C}_{\theta}(\alpha)=\left\{\theta_{0}: \theta_{0}^{\prime} \bar{A} \theta_{0}+\bar{b}^{\prime} \theta_{0}+0.63 \leq 0\right\}$, where

$$
A=\left(\begin{array}{ccc}
0.7 & 6.17 & 87.34 \\
6.14 & 170.88 & 3210.82 \\
87.34 & 3210.82 & 61730.62
\end{array}\right), \bar{A}=\left(\begin{array}{ccc}
770.72 & -770.70 & -13287.73 \\
-770.70 & 770.72 & 13287.70 \\
-13287.73 & 13287.70 & 270277.74
\end{array}\right)
$$

$b=(-0.8,-15.62,-285.9)^{\prime}$ and $\bar{b}=(-33.59,33.59,838.17)^{\prime}$.The matrix $A$ has exactly one negative eigenvalue, while all eigenvalues of $\bar{A}$ are positive. Hence, $\mathscr{C}_{\beta}(\alpha)$ is an unbounded ellipsoid, while $\mathscr{C}_{\theta}(\alpha)$ is a bounded ellipsoid, thus confirming that $\theta$ is identified while $\beta$ is not. Then, for any scalar linear transformations $w^{\prime} \theta$, a confidence set with level $1-\alpha_{2}$ is given by (3.16) with $\hat{\theta}=$ $(0.279,0.312,-0.003)$ and $\bar{D}\left(\alpha_{2}\right)=0.72\left[w^{\prime}\left(Y^{\prime} M Y\right)^{-1} w\right]^{1 / 2}$. For $w^{\prime} \beta$, we can obtain a projectionbased confidence set with level $1-\alpha_{1}$ by using (3.21) with $\tilde{\beta}=(-0.361,0.218,-0.010)$, $d=-1.55<0$ and $D\left(\alpha_{1}\right)=\left[-1.55 w^{\prime} A^{-1} w\right]^{1 / 2}$ when $w^{\prime} A^{-1} w<0$. For inference on $a$, we also use the following estimates:

$$
\hat{a}=\left(\begin{array}{c}
-0.102 \\
0.102 \\
-0.004
\end{array}\right), \hat{\sigma}_{V u}=\left(\begin{array}{c}
-0.492 \\
0.492 \\
7.634
\end{array}\right), \hat{\Sigma}_{V}=\left(\begin{array}{ccc}
3.76 & -3.75 & -64.75 \\
-3.75 & 3.74 & 64.76 \\
-64.75 & 64.76 & 1317.14
\end{array}\right) .
$$

The 2SLS estimate of $\beta$ is $\hat{\beta}_{2 S L S}=(0.190,0.019,0.001)^{\prime}$, and the eigenvalues of $\hat{\Pi}_{2}^{\prime} \hat{\Pi}_{2}$, where $\hat{\Pi}_{2}$ is the OLS estimate of $\Pi_{2}$ from (5.6), are: $(0.0003,0.095,3858.326)$. The value 0.0003 is quite 
Table 2. Card model of education and earnings

Projection-based confidence intervals for endogeneity parameters (95\% level)

\begin{tabular}{|c|c|c|c|}
\hline \multicolumn{3}{|c|}{ Without $I Q$ variable } \\
\hline \multicolumn{3}{|c|}{ Regression endogeneity } & \multicolumn{2}{|c|}{ Covariance endogeneity } \\
\hline $\mathscr{C}_{a_{1}}$ & ]$-\infty, 0.47] \cup[1.45,+\infty[$ & $\mathscr{C}_{\sigma_{V u 1}}$ & ]$-\infty, 0.41] \cup[9.08,+\infty[$ \\
$\mathscr{C}_{a_{2}}$ & ]$-\infty,-0.12] \cup[-0.03,+\infty[$ & $\mathscr{C}_{\sigma_{V u 3}}$ & ]$-\infty,-9.08] \cup[-0.41,+\infty[$ \\
$\mathscr{C}_{a_{3}}$ & ]$-\infty, 0.002] \cup[0.03,+\infty[$ & $\mathscr{C}_{\sigma_{V u 3}}$ & ]$-\infty,-165.35] \cup[-7.65,+\infty[$ \\
\hline \multicolumn{3}{|c|}{ With $I Q$ variable } \\
\hline $\mathscr{C}_{a_{1}}$ & ]$-\infty, 0.55] \cup[0.73,+\infty[$ & $\mathscr{C}_{\sigma_{V u 1}}$ & ]$-\infty, 0.24] \cup[3.19,+\infty[$ \\
$\mathscr{C}_{a_{2}}$ & $\mathbb{R}$ & $\mathscr{C}_{\sigma_{V u 3}}$ & ]$-\infty,-3.19] \cup[-0.24,+\infty[$ \\
$\mathscr{C}_{a_{3}}$ & ]$-\infty, 0.001] \cup[0.013,+\infty[$ & $\mathscr{C}_{\sigma_{V u 3}}$ & ]$-\infty,-52.05] \cup[-4.37,+\infty[$ \\
\hline
\end{tabular}

close to zero, which suggests instruments are weak. ${ }^{7}$

Table 2 presents the projection-based confidence intervals with level 95\% for individual components of endogeneity parameters $\left(a\right.$ and $\sigma_{V u}$ ). In the first part of the table, the $I Q$ variable is omitted from the model, and it is included in the second part. The results are similar with and without this variable: the confidence intervals for all components of $a$ and $\sigma_{V u}$ are unbounded. So, all components of both endogeneity parameters are weakly identified. While the estimate of $a_{3}\left(\hat{a}_{3}=-0.004\right)$ seems very close to zero, the corresponding covariance estimate $\hat{\sigma}_{V u 3}=7.634$ is relatively large, which confirms the fact that $a_{i}=0$ does necessarily not implies that $\sigma_{V u i}=0$, as argued in Section 2.1. All confidence intervals, thought unbounded, contain zero, suggesting that there is not enough information from the data to: (1) support the presence of bias due to omitted variables (regression endogeneity parameters $a_{i}, i=1,2,3$, measure the importance of omitted variables), and (2) reject the partial exogeneity of the schooling and experience variables (covariance endogeneity parameters $\sigma_{V u i}, i=1,2,3$, measure the endogeneity of the corresponding variable $Y_{i}$ ). Meanwhile, though zero belongs to the $95 \%$ confidence intervals of all these parameters, it may be the case that the true values of these parameters are actually large, because the the $95 \%$ corresponding confidence intervals are unbounded. So, the use of the standard $t$-type statistics based on the estimates of $a$ and $\sigma_{V u}$ in the extended regression (2.14), where $V$ is replace by $\hat{V}=M Y$, to build confidence intervals for scalar linear transformations $w^{\prime} a$ and $w^{\prime} \sigma_{V u}$ can be misleading when identification is weak. The Monte Carlo simulations indicate that such $t$-type confidence intervals have poor coverage probabilities (which may even be equal to zero) when identification is weak, while the coverage probabilities of the projection method developed in this paper are always above $1-\alpha$ irrespective of whether identification is strong or weak, where $\alpha$ is the nominal level.

\footnotetext{
${ }^{7}$ The results reported are based on the critical values of the $F$-distributions of Section 3 . The Monte Carlo method as described in Section 3.5 gives similar results even with 1000 replications, for both (1) Gaussian errors, and (2) Studenttype errors with three degrees of freedom.
} 


\section{Conclusion}

In this paper, we have studied the problem of testing hypotheses and building confidence sets on endogeneity parameters. Such parameters have both intrinsic and statistical interest, because they represent the effect of "common factors" which induce simultaneity and determine simultaneity biases (along with other features of the data). We stressed the usefulness of distinguishing between regression endogeneity parameters $(a)$ and covariance endogeneity parameters $\left(\sigma_{V u}\right)$ : regression endogeneity parameters measure the effect of "missing variables" in linear structural equations, while covariance endogeneity parameters directly indicate which variables may be treated as "exogenous" in statistical inference. Further, regression endogeneity parameters may be tested relatively easily, and we proposed finite-sample inference methods for these. Inference on covariance endogeneity parameters involves additional nuisance parameters (e.g., the unknown covariance matrix $\Sigma_{V}$ ), so only asymptotically justified methods were given for $\sigma_{V u}$.

The identification of endogeneity parameters was also discussed. After formulating necessary and sufficient conditions for the identification of such parameters, we observed a simple equivalence between the identification of individual regression endogeneity parameters $\left(a_{i}\right)$ and the identification of the corresponding structural parameters $\left(\beta_{i}\right)$, while this feature does not hold for covariance endogeneity parameters. In view of the possibility of identification failure, identification-robust inference procedures were proposed for endogeneity parameters. For joint hypotheses involving structural and regression endogeneity parameters, as well as marginal hypotheses on regression endogeneity parameters, finite-sample procedures were proposed. Under Gaussian errors, the tests and confidence sets are based on standard Fisher critical values. For a wide class of parametric nonGaussian errors (possibly heavy-tailed), exact Monte Carlo procedures can be applied using the statistics considered. As a special case, this result also holds for usual AR-type tests and confidence sets on structural coefficients.

We showed that the proposed finite-sample procedures (e.g., those based on a Gaussian assumption on the errors) remain asymptotically valid under weaker distributional assumptions. Tests of partial exogeneity hypotheses (for individual potentially endogenous explanatory variables) are covered as instances of the class of proposed procedures. The asymptotic theory also yields inference for covariance endogeneity. Even though the asymptotic theory is only approximate in finite samples, it is robust to identification assumptions. Finally, the proposed procedures were applied to two empirical examples: the relation between trade and economic growth, and the widely studied problem of returns to education. 


\section{APPENDIX}

\section{A. Proof}

Proof of Proposition 3.1 On multiplying the two sides of (3.2) by $M$ and $M_{1}-M$, we see that:

$$
\begin{aligned}
M\left(y-Y \beta_{0}\right) & =M u+M V\left(\beta-\beta_{0}\right) \\
\left(M_{1}-M\right)\left(y-Y \beta_{0}\right) & =M_{1} X_{2} \Pi_{2}\left(\beta-\beta_{0}\right)+\left(M_{1}-M\right) u+\left(M_{1}-M\right) V\left(\beta-\beta_{0}\right) .
\end{aligned}
$$

When Assumption 3.1 holds and $\beta=\beta_{0}$, this entails:

$$
M\left(y-Y \beta_{0}\right)=\sigma(X) M v,\left(M_{1}-M\right)\left(y-Y \beta_{0}\right)=\sigma(X)\left(M_{1}-M\right) v .
$$

Thus, the $A R$-statistic in (3.3) can be rewritten as:

$$
A R\left(\beta_{0}\right)=\frac{\sigma(X)^{2} v^{\prime}\left(M_{1}-M\right) v / k_{2}}{\sigma(X)^{2} v^{\prime} M v /(T-k)}=\frac{v^{\prime}\left(M_{1}-M\right) v / k_{2}}{v^{\prime} M v /(T-k)} .
$$

Hence, the null conditional distribution of $A R\left(\beta_{0}\right)$, given $X$, only depends on $v$ and $X$. If normality holds conditional on $X$, i.e. $v \mid X \sim \mathrm{N}\left[0, I_{T}\right]$, we have $v^{\prime} M v \sim \chi^{2}(T-k)$ and $v^{\prime}\left(M_{1}-M\right) v \sim$ $\chi^{2}\left(k_{2}\right)$. Since $M\left(M_{1}-M\right)=0$, hence $v^{\prime} M v$ and $v^{\prime}\left(M_{1}-M\right) v$ are independent conditional on $X$. Consequently, $A R\left(\beta_{0}\right) \sim F\left(k_{2}, T-k\right)$. 


\section{References}

Abdelkhalek, T., Dufour, J.-M., 1998. Statistical inference for computable general equilibrium models with applications to a model of the Moroccan economy. Review of Economics and Statistics, pp. 520-534.

Ahn, S., 1997. Orthogonality tests in linear models. Oxford Bulletin of Economics and Statistics $59,83-186$.

Anderson, T. W., Rubin, H., 1949. Estimation of the parameters of a single equation in a complete system of stochastic equations. Annals of Mathematical Statistics 20, 46-63.

Angrist, J. D., Krueger, A. B., 1991. Does compulsory school attendance affect schooling and earning?. Quarterly Journal of Economics CVI, 979-1014.

Barro, R., 1977. Unanticipated money growth and unemployment in the United States. American Economic Review 67, 101-115.

Baum, C., Schaffer, M., Stillman, S., 2003. Instrumental variables and GMM: Estimation and testing. 3(1), 1-30.

Bound, J., Jaeger, D. A., Baker, R. M., 1995. Problems with instrumental variables estimation when the correlation between the instruments and the endogenous explanatory variable is weak. Journal of the American Statistical Association 90, 443-450.

Card, D., 1995. Using geographic variation in college proximity to estimate the return to schooling. In: D. Card, ed., Aspects of Labour Market Behaviour: Essays in Honour of John Vanderkamp. University of Toronto Press: in L. N. Christo. des, E. K. Grant, and R. Swidinsky Eds, Toronto, Canada.

Chmelarova, V., Hill, R., 2010. The Hausman pretest estimator. Economics Letters 108, 96-99.

Davidson, R., Godfrey, L., Mackinnon, J. G., 1985. A simplified version of the differencing test. International Economic Review 26(3), 639-647.

Davidson, R., Mackinnon, J., 1993. Econometric Theory and Methods. Oxford University Press, New York, New York.

Davidson, R., Mackinnon, J. G., 1985. The interpretation of test statistics. Canadian Journal of Economics 18(1), 38-57.

Davidson, R., Mackinnon, J. G., 1989. Testing for consistency using artificial regressions. Econometric Theory 5(3), 363-384.

Davidson, R., Mackinnon, J. G., 1990. Specification tests based on artificial regressions. Journal of the American Statistical Association 85(409), 220-227. 
Doko Tchatoka, F., Dufour, J.-M., 2011a. Exogeneity tests and estimation in IV regressions. Technical report, Department of Economics, McGill University, Canada Montréal, Canada.

Doko Tchatoka, F., Dufour, J.-M., 2011b. On the finite-sample theory of exogeneity tests with possibly non-Gaussian errors and weak identification. Technical report, Department of Economics, McGill University, Canada Montréal, Canada.

Dufour, J.-M., 1979. Methods for Specification Errors Analysis with Macroeconomic Applications $\mathrm{PhD}$ thesis University of Chicago. $257+$ XIV pages.

Dufour, J.-M., 1987. Linear Wald methods for inference on covariances and weak exogeneity tests in structural equations. In: I. B. MacNeill, G. J. Umphrey, eds, Advances in the Statistical Sciences: Festschrift in Honour of Professor V.M. Joshi's 70th Birthday. Volume III, Time Series and Econometric Modelling. D. Reidel, Dordrecht, The Netherlands, pp. 317-338.

Dufour, J.-M., 1990. Exact tests and confidence sets in linear regressions with autocorrelated errors. Econometrica 58, 475-494.

Dufour, J.-M., 1997. Some impossibility theorems in econometrics, with applications to structural and dynamic models. Econometrica 65, 1365-1389.

Dufour, J.-M., 2003. Identification, weak instruments and statistical inference in econometrics. Canadian Journal of Economics 36(4), 767-808.

Dufour, J.-M., 2006. Monte carlo tests with nuisance parameters: A general approach to finitesample inference and nonstandard asymptotics in econometrics. Journal of Econometrics $138,2649-2661$.

Dufour, J.-M., Hsiao, C., 2008. Identification. In: L. E. Blume, S. N. Durlauf, eds, The New Palgrave Dictionary of Economics second edn. Palgrave Macmillan, Basingstoke, Hampshire, England. forthcoming.

Dufour, J.-M., Jasiak, J., 2001. Finite sample limited information inference methods for structural equations and models with generated regressors. International Economic Review 42, 815-843.

Dufour, J.-M., Taamouti, M., 2005. Projection-based statistical inference in linear structural models with possibly weak instruments. Econometrica 73(4), 1351-1365.

Dufour, J.-M., Taamouti, M., 2007. Further results on projection-based inference in IV regressions with weak, collinear or missing instruments. Journal of Econometrics 139(1), 133-153.

Durbin, J., 1954. Errors in variables. Review of the International Statistical Institute 22, 23-32.

Engle, R. F., 1982. A general approach to Lagrange multiplier diagnostics. Journal of Econometrics 20, 83-104.

Farebrother, R. W., 1976. A remark on the Wu test. Econometrica 44, 475-477. 
Frankel, J. A., Romer, D., 1999. Does trade cause growth?. American Economic Review 89(3), 379399.

Hahn, J., Ham, J., Moon, H. R., 2010. The Hausman test and weak instruments. Journal of Econometrics 160, 289-299.

Hausman, J., 1978. Specification tests in econometrics. Econometrica 46, 1251-1272.

Hausman, J., Taylor, W. E., 1981. A generalized specification test. Economics Letters 8, 239-245.

Holly, A., 1982. A remark on Hausman's test. Econometrica 50, 749-759.

Holly, A., 1983a. Tests d'exogénéité dans un modèle à equations simultanées: Énoncé de résultats théoriques en information limitée et illustrations à des tests de dépendance de la politique monétaire en régime de changes fixes. Cahiers du Séminaire d'Économétrie 25, 49-69.

Holly, A., 1983b. Une présentation unifiée des tests d'exogénéity dans les modèles à equations simultanées. Annales de l'INSEE 50, 3-24.

Hwang, H.-S., 1980. Test of independence between a subset of stochastic regressors and disturbances. International Economic Review 21, 749-760.

Hwang, H.-S., 1985. The equivalence of Hausman and Lagrange multiplier tests of independence between disturbance and a subset of stochastic regressors. Economics Letters 17, 83-86.

Jeong, J., Yoon, B. H., 2010. The effect of pseudo-exogenous instrumental variables on hausman test. Communications in Statistics, Simulation and Computation 39(2), 315-321.

Kariya, T., Hodoshima, H., 1980. Finite-sample properties of the tests for independence in structural systems and LRT. The Quarterly Journal of Economics 31, 45-56.

Kiviet, J. F., 2013. Identification and inference in a simultaneous equation under alternative information sets and sampling schemes. The Econometrics Journal 16, S24-S59.

Kiviet, J. F., Niemczyk, J., 2007. The asymptotic and finite-sample distributions of OLS and simple IV in simultaneous equations. Computational Statistics and Data Analysis 51, 3296-3318.

Kiviet, J. F., Niemczyk, J., 2012. Comparing the asymptotic and empirical (un)conditional distributions of OLS and IV in a linear static simultaneous equation. Computational Statistics and Data Analysis 56, 3567-3586.

Kiviet, J. F., Pleus, M., 2012. The performance of tests on endogeneity of subsets of explanatory variables scanned by simulation. Technical report, Amsterdam, The Netherlands.

Kleibergen, F., 2002. Pivotal statistics for testing structural parameters in instrumental variables regression. Econometrica 70(5), 1781-1803.

Kleibergen, F., 2004. Testing subsets of structural coefficients in the IV regression model. Review of Economics and Statistics 86, 418-423. 
Meepagala, G., 1992. On the finite sample performance of exogeneity tests of Revankar, Revankar and Hartley and Wu-Hausman. Econometric Reviews 11, 337-353.

Moreira, M. J., 2003. A conditional likelihood ratio test for structural models. Econometrica 71(4), 1027-1048.

Nakamura, A., Nakamura, M., 1981. On the relationships among several specification error tests presented by Durbin, Wu and Hausman. Econometrica 49, 1583-1588.

Nakamura, A., Nakamura, M., 1985. On the performance of tests by Wu and by Hausman for detecting the ordinary least squares bias problem. Journal of Econometrics 29, 213-227.

Newey, W. K., 1985a. Generalized method of moments specification testing. Journal of Econometrics $29,229-256$.

Newey, W. K., 1985b. Maximum likelihood specification testing and conditional moment tests. Econometrica 53(5), 1047-1070.

Revankar, N. S., 1978. Asymptotic relative efficiency analysis of certain tests in structural sysytems. International Economic Review 19, 165-179.

Revankar, N. S., Hartley, M. J., 1973. An independence test and conditional unbiased predictions in the context of simultaneous equation systems. International Economic Review 14, 625-631.

Ruud, P. A., 1984. Tests of specification in econometrics. Econometric Reviews 3(2), 211-242.

Ruud, P. A., 2000. An Introduction to Classical Econometric Theory. Oxford University Press, Inc., New York.

Smith, R. J., 1983. On the classical nature of the Wu-Hausman statistics for independence of stochastic regressors and disturbance. Economics Letters 11, 357-364.

Smith, R. J., 1984. A note on likelihood ratio tests for the independence between a subset of stochastic regressors and disturbances. International Economic Review 25, 263-269.

Smith, R. J., 1985. Wald tests for the independence of stochastic variables and disturbance of a single linear stochastic simultaneous equation. Economics Letters 17, 87-90.

Smith, R. J., Pesaran, M., 1990. A unified approach to estimation and orthogonality tests in linear single-equation econometric models. Journal of Econometrics 44, 41-66.

Spencer, D. E., Berk, K. N., 1981. A limited-information specification test. Econometrica 49, 10791085. Erratum, Econometrica, Vol. 50, No. 4 (Jul., 1982), p. 1087.

Staiger, D., Stock, J. H., 1997. Instrumental variables regression with weak instruments. Econometrica $65(3), 557-586$.

Stock, J. H., Wright, J. H., Yogo, M., 2002. A survey of weak instruments and weak identification in generalized method of moments. Journal of Business and Economic Statistics 20(4), 518-529. 
Thurman, W., 1986. Endogeneity testing in a supply and demand framework. Review of Economics and Statistics 68(4), 638-646.

Wong, K.-f., 1996. Bootstrapping Hausman's exogeneity test. Economics Letters 53, 139-143.

Wu, D.-M., 1973. Alternative tests of independence between stochastic regressors and disturbances. Econometrica 41, 733-750.

Wu, D.-M., 1974. Alternative tests of independence between stochastic regressors and disturbances: Finite sample results. Econometrica 42, 529-546.

Wu, D.-M., 1983a. A remark on a generalized specification test. Economics Letters 11, 365-370.

Wu, D.-M., 1983b. Tests of causality, predeterminedness and exogeneity. International Economic Review 24(3), 547-558. 